\section{Constrained Bearings-Only Target Motion Analysis via Markov Chain Monte Carlo Methods}

\author{
FRÉDÉRIC BAVENCOFF \\ JEAN-MICHEL VANPEPERSTRAETE \\ Thales Airborne Systems \\ France \\ J.-PIERRE LE CADRE, Member IEEE \\ IRISA/CNRS \\ France
}

The aim of this paper is to develop methods for estimating the range of a moving target from bearings-only observations and for weakly observable scenarios, by including general constraints about the target trajectories. Throughout this manuscript, it is assumed that the target motion is conditionally deterministic, which leads us to focus on batch algorithms. Another common assumption is poor observability, which results from another constraint: (very) limited amplitude of the observer maneuvers. Classical batch algorithms are based on iterative methods (such as gradient techniques), but they perform very poorly in this context and including constraints is not easy and not reliable in this way.

Instead, we consider simulation-based methods, i.e., Monte Carlo Markov chain (MCMC) sampling, developed in a Bayesian context. In this way, it is possible to take into account any type of constraint, and to drastically improve estimation of weakly observable parameters. As a by-product, an estimate of confidence intervals is obtained. This is the aim of the highest probability density (HPD) Intervals. This study is illustrated by simulation results, while the benefits of constraint inclusion are analyzed via geometric methods.

Manuscript received March 9, 2004; revised March 23, 2005 and January 11, 2006; released for publication February 24, 2006

IEEE Log No. T-AES/42/4/890176.

Refereeing of this contribution was handled by D. J. Salmond.

Authors' addresses: F. Bavencoff and J-M. Vanpeperstraete, Thales Airborne Systems, 2 Avenue Gay-Lussac, 78851, Elancourt, France; J.-P. Le Cadre, IRISA/CNRS, Campus de Beaulieu, 35042, Rennes (Cedex), France, E-mail: (lecadre@ irisa.fr).

0018-9251/06/\$17.00 (c) 2006 IEEE

\section{INTRODUCTION}

The general context we consider here is that of bearings-only $\left(\mathrm{BOT}^{1}\right)$ target motion analysis (TMA). More precisely, noisy bearings collected by a single receiver are the only available observations. It is perhaps in the passive sonar environment that BOT TMA is most familiar; though its relevance is growing rapidly for other contexts such as surface and airborne platforms (I. R. sensors) or passive surveillance via electronic support measurement (ESM). To illustrate the aim of this article, we quote the following extract from [42]. "In bearings-only TMA, particular importance is attached to range estimation. Target position is the prime determinant in deciding whether to fire a weapon, to commence evasion, or to continue pursuit." This clearly motivates this article whose major objective is range estimation for "difficult" scenarios. The difficulty here is related to a poor observability, itself resulting from operational requirements including limited observer maneuverability.

Let us now summarize the observer constraints. While it is generally recognized [30] that observer maneuvers is the basic ingredient for BOT TMA; both maneuver amplitude and frequency are very limited in a multi-target surveillance scenario. This is due to operational considerations and this will constitute the general scenario under consideration here. Moreover, we shall restrict attention to a two-dimensional BOT problem: the observer is confined to a plane which includes the target.

Analysis of BOT TMA is generally considered in two ways: observability $[25,20]$ and estimability $[28,27,21]$. Observability is of an algebraic nature [32], with a binary answer (yes or not). Observability is the ability to recover the target state (or equivalently its trajectory) from the (exact $\left.{ }^{2}\right)$ observations [32]. The "basic" TMA problem is (generally) observable as soon as the observer does a maneuver, e.g. makes a turn. Estimability concerns the estimation of the state vector. A problem can be just observable but have to unacceptable estimation performance.

The BOT TMA problem is certainly not new and can even be traced back to the work of C. F. Gauss (estimation of the orbit parameters of the Ceres asteroid) $[12,16]$. From a statistical perspective, it is a nonlinear regression problem. Since that time, it has been extensively investigated. One line of development is based on graphical or semi-graphical approaches [42]. These ideas have been extended to take into account the bearing-rate change (after a turn), producing well-recognized methods like the important Ekelund ranging or the Spiess method

\footnotetext{
${ }^{1}$ BOT means bearings-only tracking.

${ }^{2}$ Exact means unoisy here.
} 
[42]. Let us emphasize that, quite similarly to this paper, their aim is precisely range estimation [30]. However, to perform satisfactorily they need a substantial observer maneuver [42]. We address here the problem of weakly observable scenarios. To overcome this fundamental difficulty, the key is to include constraints on target motion which have been incorporated via appropriate Monte Carlo Markov chain (MCMC) methods. It is shown that speed constraints are very useful for reducing the uncertainty about the target trajectory. More precisely, uncertainty can be reduced by a factor proportional to the quotient of the target speed interval length by the observer speed.

We restrict our analysis to a special class of target motion: namely the "conditionally deterministic one." This means simply that conditional on a fixed number of parameters, the target trajectory is deterministic. In this setup, it has been recognized that the problem is fundamentally one of nonlinear regression, for which batch algorithms are a convenient framework; see [28] for a seminal contribution. A vast and rich literature has been devoted to this subject $[28,6,31]$. However, inclusion of constraints in the estimation process for BOT TMA is rarely considered $[14,19$, 29]. For instance, the use of Uzawa's like method has been mentioned in [28] and [14]. However, practically, results seem to indicate a neat tendency to "push" the solution toward the constraint bounds [33]. The reason is simple, the likelihood functional is flat relative to the unobservable component(s). So, it is appropriate to replace point estimation by interval estimation and to include state constraints in the estimation process.

To a certain extent, this is also the aim of geometric methods like "semi-azimetrie" [37, 4]; very simple in principle, but providing robust and often adequate performance. ${ }^{3}$ However, these methods are only able to accommodate simple kinematic constraints for computing intervals and do not give a useful statistical insight. They are not designed for estimating highest probability density (HPD) intervals [9] or dealing with general and complex constraints, which is precisely the objective of this work. Another fundamental reason to use MCMC methods is that marginal maximum a posteriori estimation for Bayesian models can be achieved very simply in this setting $[13,10]$. Thus, it is not surprising that its importance has been recognized also in target tracking, especially for stressing scenarios [11].

The effects of constraint inclusion on state estimation have been investigated, both from a statistical perspective (Appendix A) and a

\footnotetext{
${ }^{3}$ For a mathematical analysis of these methods we refer to
} Appendix B. deterministic one (Appendix B). Both have their own advantages. The first one has the definite advantage to take into account the statistical characteristics of the problem. However, it is fundamentally a local analysis. The aim of the deterministic investigation is to provide state parameter bounding. It is a global approach which allows us to investigate the performance of the MCMC methods, especially the effects of constraints. Geometry of nonobservable BOT TMA has been analyzed both from a nonlinear perspective (envelope geometry) and a linear one (the geometry of the observable subspace). In this setup, ghosting has been also analyzed (see Appendix B).

This paper is organized as follows. Problem formulation is given in the Section II, followed by a presentation of the MCMC method for constrained estimation in Sections III (Metropolis algorithm) and IV (hit-and-run algorithm). In these sections, the emphasis is on application to the BOT TMA context. Section V deals with the HPD estimation. Results are presented in Section VI. Finally, proofs of the main results are available in Appendices A, B, and C.

\section{Notations and Acronyms}

\begin{tabular}{|c|c|}
\hline$A^{T}$ & Transposition of the $A$ matrix \\
\hline Id & Identity matrix \\
\hline$\hat{\theta}_{1: N}$ & Set of observed bearings: $\hat{\theta}_{1}, \ldots, \hat{\theta}_{N}$ \\
\hline$L_{\hat{\theta}_{1: N}}(\mathbf{x})$ & $\begin{array}{l}\text { Likelihood of the parameter vector } \mathbf{x} \\
\text { given observations } \hat{\theta}_{1 \cdot N}\end{array}$ \\
\hline $\operatorname{Fr}(S)$ & Frontier of $S$ domain \\
\hline $\operatorname{tgt}$ & Target \\
\hline obs & Observer \\
\hline BOT & Bearings only tracking \\
\hline TMA & Target motion analysis \\
\hline MCMC & Monte Carlo Markov chains \\
\hline HPD & Highest probability density. \\
\hline
\end{tabular}

\section{PROBLEM FORMULATION}

The problem of estimating the parameters of the target trajectory under the assumption of rectilinear and uniform (target) motion from a history of noisy passive bearing measurements is considered. The general scenario is depicted in Fig. 1. It is assumed that the observer motion is rectilinear and uniform on each leg, and that the problem is planar.

Firstly, let us briefly recall the problem in Cartesian coordinates. Let $\mathbf{X}$ be the relative (target) state vector, defined by

$$
\mathbf{X}=\mathbf{X}_{\mathrm{tgt}}-\mathbf{X}_{\mathrm{obs}} \triangleq\left[r_{x}, r_{y}, v_{x}, v_{y}\right]^{T} .
$$

The discrete time equation of the relative motion takes the following form:

$$
\mathbf{X}_{k}=F \mathbf{X}_{k-1}+\mathbf{U}_{k}
$$




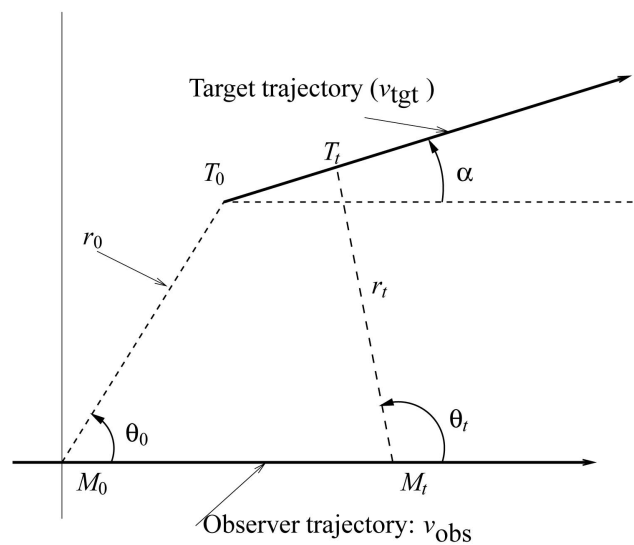

Fig. 1. BOT TMA scenario: $\theta$ : bearing; $r$ : target range; $\alpha$ : target heading; $v_{\text {tgt }}:$ target speed.

where

$$
F=\left(\begin{array}{cc}
\mathrm{Id}_{2} & \mathrm{Id}_{2} \\
0 & \mathrm{Id}_{2}
\end{array}\right) .
$$

In the above formula the vector $\mathbf{U}_{k}$ accounts for the effects of the observer accelerations, while $\mathrm{Id}_{2}$ is the two-dimensional identity matrix. Let us consider a slightly different parameterization (see Fig. 1). The initial target position is defined by its initial range $r$ and bearing $\theta$; while it is moving with a constant velocity $v$ and heading $\alpha$. The target trajectory can be also defined by the state vector $(r, \theta, v, \alpha)$, at an arbitrary reference time.

Let $\hat{\theta}_{1: N} \triangleq\left(\hat{\theta}_{1}, \ldots, \hat{\theta}_{N}\right)$ be $N$ noisy bearing measurements, at time periods $\left\{\tau_{1}, \tau_{2}, \ldots, \tau_{N}\right\}$. The bearing measurements $\hat{\theta}_{k}$ are the exact bearings corrupted by a sequence of independent and identically (normally) distributed (IID) noises. Thus, the mean ${ }^{4}$ of $\hat{\theta}_{k}$ is $\theta_{k}$, while its variance is $\sigma^{2}$. Classically, the batch BOT TMA problem is solved by a maximum likelihood estimator (MLE) which consists in finding the state vector $(r, \theta, v, \alpha)^{*}$ which maximizes the likelihood given the observed bearings $\hat{\theta}_{1: N}$, i.e.,

$$
(r, \theta, v, \alpha)^{*} \stackrel{\Delta}{\arg \max } L_{(r, \theta, v, \alpha)} L_{\hat{\theta}_{1: N}}[(r, \theta, v, \alpha)] .
$$

The likelihood of a state vector given the observed bearings is defined by the following expression:

$$
\begin{aligned}
L_{\hat{\theta}_{1: N}} & {[(r, \theta, v, \alpha)] } \\
& =f_{(r, \theta, v, \alpha)}\left(\hat{\theta}_{1: N}\right) \\
& =\left(\frac{1}{\sigma \sqrt{2 \pi}}\right)^{N} \cdot \exp \left[-\frac{1}{2 \sigma^{2}} \sum_{k=1}^{N}\left(\hat{\theta}_{k}-\theta_{k}[(r, \theta, v, \alpha)]\right)^{2}\right]
\end{aligned}
$$

\footnotetext{
${ }^{4}$ Note that the $\hat{\theta}_{1: N}$ is not identically distributed since its mean is time varying.
}

where $\theta_{k}[(r, \theta, v, \alpha)]$ is the true $k$ th angle associated with the constant velocity target trajectory defined by the state vector $(r, \theta, v, \alpha)$. With the notations of Fig. 1, we have ${ }^{5}$ (see (28)):

$$
\tan \left(\theta_{k}\right)=\frac{\sin \theta_{0}+\left(\frac{v_{\mathrm{tgt}} \sin \alpha}{r}\right) k}{\cos \theta_{0}+\left(\frac{v_{\mathrm{tgt}} \cos \alpha-v_{\mathrm{obs}}}{r}\right) k} .
$$

This parameterization of the target trajectory is chosen (instead of the Cartesian one) simply because it is more convenient for including constraints. Note, furthermore, that the parameter $\theta$ represents the target bearing at an arbitrary reference time. So, the suffix 0 $\left(\theta_{0}\right)$ is omitted. Since there does not exist an explicit method for solving (4), iterative methods are generally used for solving this nonlinear regression problem, e.g. gradient methods [28] or modified instrumental variable [28, 6] (MIV) methods. So, a vast literature has been devoted to this subject. Even if the likelihood is not concave, convergence of these methods has been analyzed [26].

However, estimability is characterized by the Cramer-Rao Lower Bound (CRLB), usually calculated within the framework of asymptotic unbiased estimators (typically MLEs). The CRLB provides a theoretical lower bound for the mean square error of the state vector estimator. It means that under regularity hypotheses, we have a (matrix) inequality

$$
\operatorname{cov}(\hat{\Theta}) \succeq I^{-1}(\Theta)
$$

where the notation $A \succeq B$ means that the $(A-B)$ matrix is semi-definite positive. $\Theta$ is the vector of the trajectory (true) parameters, while $\hat{\Theta}$ is any unbiased estimator of $\Theta$ and $\operatorname{cov}(\hat{\Theta})$ is the covariance matrix of $\hat{\Theta}$. The CRLB $I^{-1}(\Theta)$ is the inverse of the Fisher information matrix (FIM) $I(\Theta)$. Under the asymptotic efficiency assumption, the accuracy of the MLE estimator is evaluated by the CRLB. It may be shown that the MLE is asymptotically efficient in the BOT TMA context, though observations are fundamentally ${ }^{6}$ not IID [34]. Let us recall below various equivalent parameterizations for the TMA problem:

$$
\begin{cases}\mathbf{x}=\left(r_{x}, r_{y}, v_{x}, v_{y}\right)^{T} & \text { Cartesian coordinates } \\ \mathbf{x}=\left(\theta, \dot{\theta}, \frac{\dot{r}}{r}, \frac{1}{r}\right)^{T} & \text { modified polar coordinates } \\ \mathbf{x}=(r, \theta, v, \alpha)^{T} & \text { constraint coordinates. }\end{cases}
$$

In Fig. 2, we present the $r$ (diagonal) coefficient of the $I^{-1}(r, \theta, \dot{r} / r, \dot{\theta})$ matrix (i.e., the variance of $r$ ) as

${ }^{5}$ Notice that it is assumed here that the interval between measurements is constant and unit time.

${ }^{6}$ Actually, TMA is made possible only by the changes of observation density (versus time). 


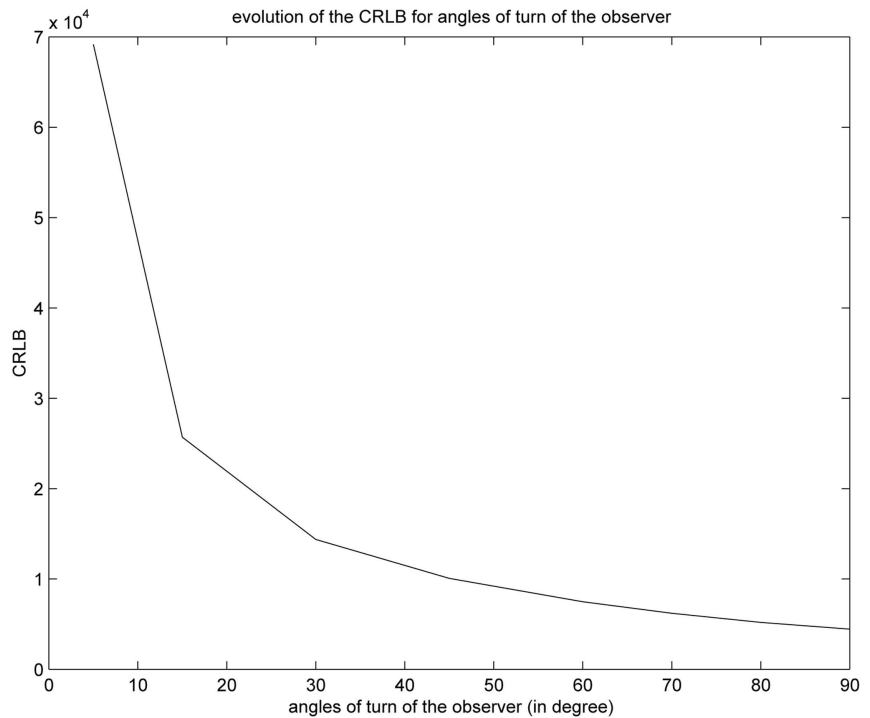

Fig. 2. Value of range CRLB (in $y$ axis) versus observer maneuver angle $\mu$.

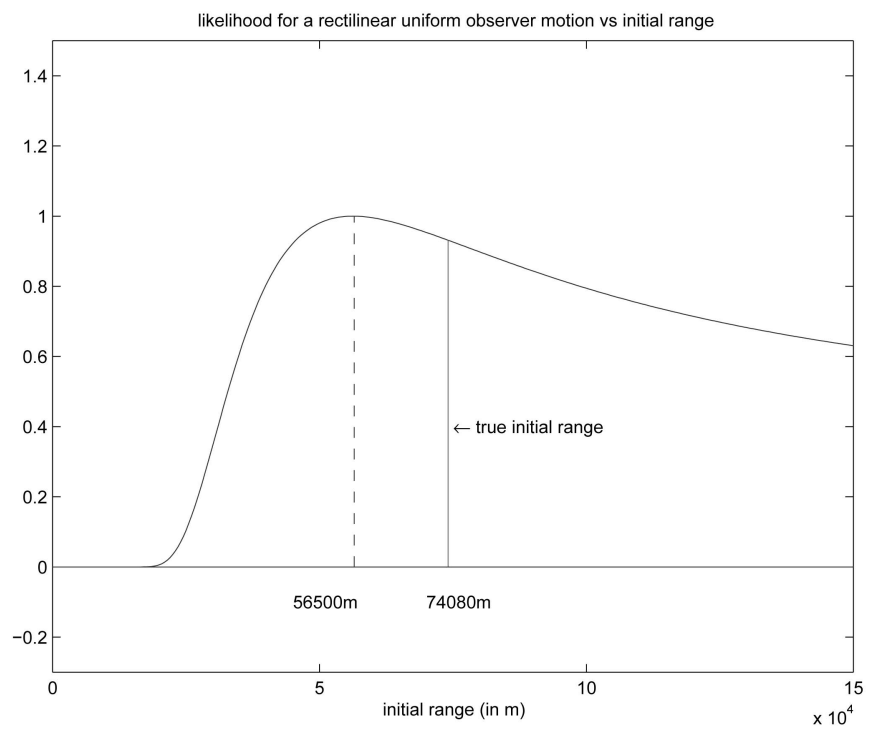

Fig. 3. Likelihood functional versus range with weak observer maneuver.

a function of the angle $\mu$ of the observer maneuver ${ }^{7}$ (moving with constant speed).

Obviously, when the amplitude of the observer maneuver is below a certain value (here, about $10 \mathrm{deg}$ ), then the $r$ CRLB coefficient grows dramatically, thus showing that the TMA problem becomes practically inestimable (see Fig. 2). If instead of heading change, we can consider a change in speed, the conclusion is similar: a very strong acceleration is necessary for estimating the range $r$. Actually, without observer maneuver, the three parameters $(\theta, \dot{r} / r, \dot{\theta})$ are observable and uncoupled with the fourth (unobservable) parameter. However, when there is an observer maneuver, full observability

\footnotetext{
${ }^{7}$ i.e., the difference of the observer headings, associated with two consecutive legs.
}

is recovered and there is an interaction between the observable part $(\theta, \dot{r} / r, \dot{\theta})$ and $r$ [1].

What is the underlying difficulty for estimating the $r$ component? It is well known that the FIM is straightforwardly related to the likelihood curvature [22]. To illustrate this dependency we present in Fig. 3 the log-likelihood versus the range $r$, the three others parameters $(\theta, \dot{r} / r, \dot{\theta})$ being fixed at their true values, the amplitude of the observer maneuver being very small. We can notice that the likelihood functional is not sharp, not symmetric and that its maximum is achieved for a false range value $(56,500 \mathrm{~m}$ versus $74,080 \mathrm{~m}$ ).

Now, it is quite common for the amplitude of the observer's maneuvers to be very limited. This may be due to operational factors, themselves related to the role of the observer itself (e.g. maritime surveillance) or to physical constraints. Indeed, it is 


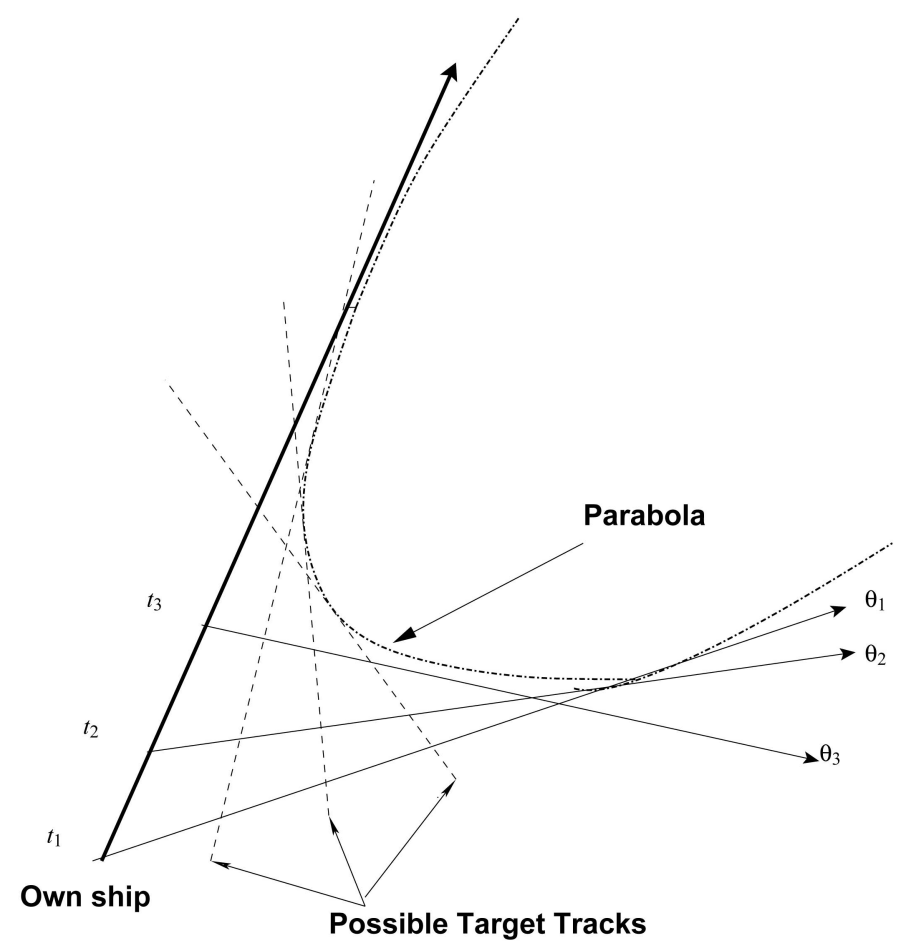

Fig. 4. Parabolic envelope of bearings and target tracks.

often impossible to tailor specific observer maneuvers to each target. So, the object of this article will be to develop reliable methods for estimating the target range within this context. To overcome the basic statistical difficulty [19] inherent in this problem (poor observability) inclusion of constraints about the target trajectory will play the principal role. To illustrate our objective, let us recall the following property taken from [42], and illustrated below ${ }^{8}$ (see Fig. 4).

PROPOSITION 1 Suppose own track is linear and distinct bearings at three times are given. A parabola is defined by those bearings and times such that all bearings are tangent to the parabola; then too are the target tracks which are consistent with the bearings.

An elementary proof of Proposition 1 is provided in the Appendix B. The aim of this article is to replace the search for the maximum of the likelihood functional by the search for an interval including the more likely values of $r$ (called HPD interval) for the low-maneuvering observer case. To compute such an interval, we assume that useful prior information is available on target speed $v$ thus narrowing the search and improving estimation of $r$. Indeed, considering Fig. 4, we see that an apparently insignificant constraint (e.g. maximal speed) can be very informative. For practical applications like maritime surveillance, operational considerations provide reasonable priors about the target speed. Let

${ }^{8}$ The interested reader can refer to the chapter 11 of [42] and its deliciously old-fashioned style.

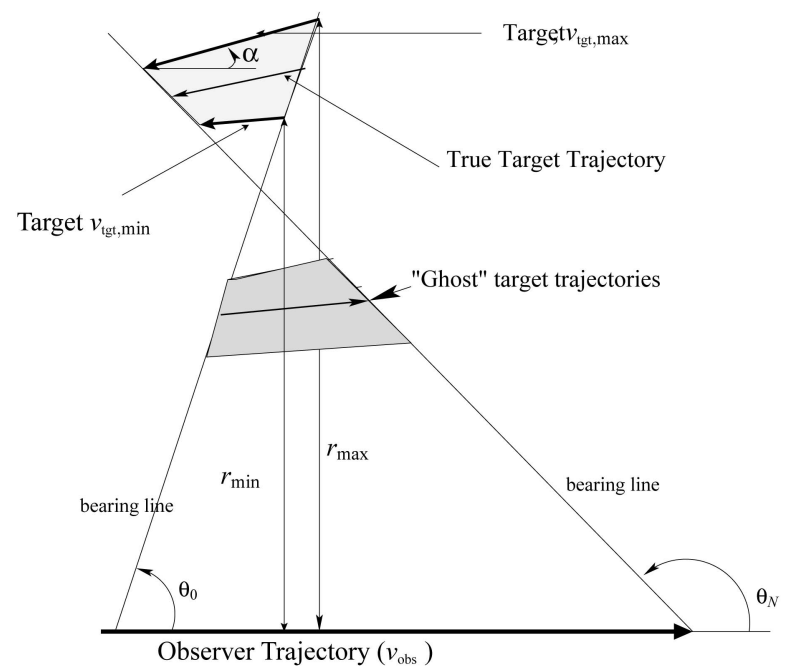

Fig. 5. Definition of uncertainty domain for Proposition 2.

us clarify this point. With the notations of Fig. 5, the following result has been obtained through elementary calculations (see Appendix B).

PROPOSITION 2 Suppose own track is linear and (exact) distinct bearings at (at last) three times are given; then with the notations of Fig. 5, we have

$$
\begin{aligned}
r_{\max }^{2}-r_{\min }^{2} & =\frac{\left(v_{\max }^{2}-v_{\text {min }}^{2}\right)}{v^{2}} r^{2} \\
& \approx 2\left[\frac{\left(v_{\text {tgt,max }}-v_{\text {tgt, } \min }\right)}{v_{\text {obs }}}\right] \cos (\alpha) r^{2} .
\end{aligned}
$$


The ratio $2\left[\left(v_{\text {tgt,max }}-v_{\text {tgt,min }}\right) / v_{\text {obs }}\right] \cos (\alpha)$ acts as a "reduction" factor for estimating the distance $r$, under speed constraints; thus showing that the benefit of including hard constraints for range estimation is generally quite noticeable.

A more intuitive representation of this phenomenon can be found in Fig. 5. For a given (target) speed constraint $\left(v_{\mathrm{tgt}, \min } \leq v_{\mathrm{tgt}} \leq v_{\mathrm{tgt} \text { max }}\right)$, admissible target trajectories are inside the two shaded areas. One area corresponds to trajectories forming an acute angle (close to zero) with the true target trajectory, while the the other (ghost) corresponds to obtuse angles (close to $\pi$ ). So, they are named "ghost" target trajectories. Obviously, this reduction factor is really interesting when the observer speed largely exceeds the target one. Therefore, the key idea consists in incorporating prior information, as constraints on target speed. In the first case, constraints are defined by intervals and used in the Metropolis MCMC algorithm. Then (Section IV), constraints are defined in terms of the target trajectory itself, for which the hit-an-run MCMC is especially relevant. This is summarized below:

$$
\begin{cases}\text { Constraints: } & \text { target speed, viable domain, } \\
\text { Target motion: } & \begin{array}{l}
\text { rectilinear or piecewise linear } \\
\text { (leg-by-leg) }
\end{array}\end{cases}
$$

It is assumed that target speed is constant on each leg.

\section{CONSTRAINTS ON TARGET SPEED GIVEN AS INTERVAL}

Prior information can be integrated in a Bayesian context. Given observations $\left(\hat{\theta}_{1: N} \triangleq\left\{\hat{\theta}_{1}, \ldots, \hat{\theta}_{N}\right\}\right.$, the posterior distribution of the state vector is given as the product of the prior with the likelihood function via the Bayes's theorem:

$$
\pi\left[(r, \theta, v, \alpha) \mid \hat{\theta}_{1: N}\right]=\frac{L_{\hat{\theta}_{1: N}}(r, \theta, v, \alpha) \times \pi(r, \theta, v, \alpha)}{\pi\left(\hat{\theta}_{1: N}\right)}
$$

where

$$
\pi\left[(r, \theta, v, \alpha) \mid \hat{\theta}_{1: N}\right] \text { is the posterior density of the }
$$
state vector,

$\pi(r, \theta, v, \alpha)$ is the prior density of the state vector,

$L_{\hat{\theta}_{1: N}}(r, \theta, v, \alpha)$ is the likelihood of the state vector $(r, \theta, v, \alpha)$ given the observations,

$\pi\left(\hat{\theta}_{1: N}\right)$ is the density of the observations.

For determining an interval for range $r$, given a prior, it is necessary to access to the marginal posterior distribution $\pi\left(r \mid \hat{\theta}_{1: N}\right)$ of the posterior density state vector $\pi\left[(r, \theta, v, \alpha) \mid \hat{\theta}_{1: N}\right]$. The main difficulty lies in the fact that the marginal posterior density $\pi\left(r \mid \hat{\theta}_{1: N}\right)$

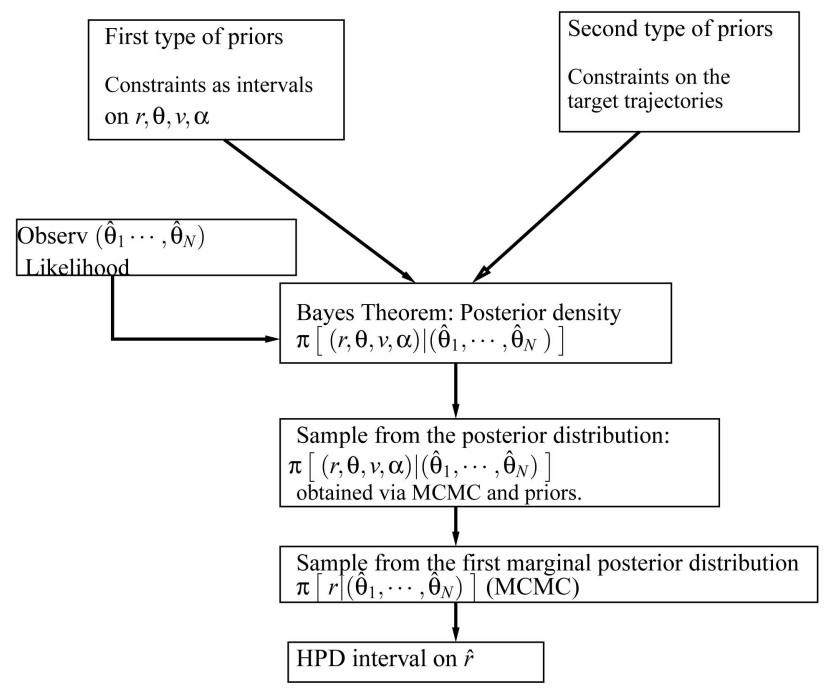

Fig. 6. General architecture for sampling, marginalization, and estimation of confidence intervals.

is defined by the integral of the posterior density $\pi\left[(r, \theta, v, \alpha) \mid \hat{\theta}_{1: N}\right]$, according to

$$
\pi\left(r \mid \hat{\theta}_{1: N}\right)=\int \pi\left[(r, \theta, v, \alpha) \mid \hat{\theta}_{1: N}\right] d(\theta, v, \alpha) .
$$

Since this may be a very demanding calculation, we shall use a different approach for approximating this posterior density based on simulation. The strength of this method is also to produce confidence intervals for constrained estimation of $r$ based on HPD intervals.

Roughly, an HPD interval is a confidence interval of minimum length, for a given probability of content (see Section V). For a given probability content, the HPD method provides a confidence interval for the range $r$ by using a sample $\left\{r^{(i)}\right\}_{i=1: n}$ from the marginal posterior density $\pi\left(r \mid \hat{\theta}_{1: N}\right)$. This is obtained by taking the first component of a sample $\left\{(r, \theta, v, \alpha)^{(i)}\right\}_{i=1: n}$, itself generated from the posterior distribution $\pi\left[(r, \theta, v, \alpha) \mid \hat{\theta}_{1: N}\right]$ via MCMC sampling, as summarized in Fig. 6.

\section{A. Prior Modelling}

As will be shown, taking into account prior information on the target speed $(v)$ is instrumental in this solution. For instance, lower and upper bounds $\left(v_{\min }\right.$ and $\left.v_{\max }\right)$ are considered. Other priors are relative to the parameters $r, \theta$, and $\alpha$. Thus, it is natural to assume lower and upper bounds for range $r$ (namely $r_{\min }$ and $r_{\max }$ ). ${ }^{9}$ Similarly, we can suppose that the first bearing measured $\hat{\theta}_{1}$ is not too far from the right bearing $\theta_{1}$, i.e., in the interval $\left[\hat{\theta}_{1}-m \sigma, \hat{\theta}_{1}+m \sigma\right]$ where $\sigma$ is the standard deviation of

${ }^{9}$ From an operational point of view, detecting measurements coming from a very remote target is not likely and has no operational interest. 
the measurements. Typically, a value $m=5$ is largely sufficient. The target heading $\alpha$ is let free in its own interval $[-\pi,+\pi]$.

In the absence of prior about the kinematic parameter densities and for simplifying the sampling process, we avoid too specific prior, so that uniform distributions are the more convenient: ${ }^{10}$

$$
\begin{array}{rlrl}
\pi(r) & \sim U\left[r_{\min }, r_{\max }\right], & & \pi(\theta) \sim U\left[\hat{\theta}_{1}-m \sigma, \hat{\theta}_{1}+m \sigma\right] \\
\pi(v) & \sim U\left[v_{\min }, v_{\max }\right], & \pi(\alpha) \sim U[-\pi,+\pi] .
\end{array}
$$

so, that (independence of the prior):

$$
\begin{aligned}
\pi(r, \theta, v, \alpha) & =\pi(r) \times \pi(\theta) \times \pi(v) \times \pi(\alpha), \\
& =C \mathbf{1}_{\left[r_{\min }, r_{\max }\right]}(r) \times \mathbf{1}_{\left[\theta_{\min }, \theta_{\max }\right]}(\theta) \times \mathbf{1}_{\left[v_{\min }, v_{\max }\right]}
\end{aligned}
$$

where $C=1 /\left(4 m \pi \sigma\left(r_{\max }-r_{\min }\right)\left(v_{\max }-v_{\min }\right)\right)$, and while 1 is the indicator function. Note that the great interest $(r, \theta, v, \alpha)$ parameterization is precisely to render explicit operational constraints.

B. Generation of Multidimensional Random Variables. The MCMC Metropolis-Hastings Algorithm

The aim of this section is to provide a specific setting for the application of the MCMC methods [18] for constrained estimation [41]. In this part, the only constraint deals with target speed and priors are defined as intervals on parameters $r, \theta, v$, and $\alpha$.

In this setup, an MCMC method is used for drawing a sample $\left\{X_{1}, X_{2}, \ldots, X_{n}\right\}$ approximately distributed from $f$ without directly simulating from $f$ (see [35]). More precisely, its aim is to produce an ergodic Markov chain $\left(X^{(t)}\right)$ with stationary distribution $f$, i.e., $\left(X^{(t)}\right)$ converges in distribution to a random variable drawn from $f$. For convergence analysis, we refer to [17]. In our context, the stationary distribution is the posterior distribution of the target trajectory $\pi\left[(r, \theta, v, \alpha) \mid \hat{\theta}_{1: N}\right]$.

For an arbitrary starting value $x^{(0)}$, a chain $\left(X^{(t)}\right)$ is generated using a transition kernel with stationary distribution $f$. Thus, for a large enough $T_{0}, X^{\left(T_{0}\right)}$ the method produces a dependent sample $X^{T_{0}}, X^{T_{0}+1}, \ldots$ which is generated from $f$.

A symmetric conditional density $q(y \mid x)$ (transition kernel) is then chosen ("symmetric" means that $q(y \mid x)=q(x \mid y))$, which defines the density to move from a state $x$ to a state $y$. The transition kernel must allow every point in the support of the density $f$ to be reached. The Metropolis-Hastings algorithm associated with the objective target density $f$ and the conditional density $q$ produces a Markov chain $\left(X^{(i)}\right)$ through the following transition.

\footnotetext{
${ }^{10}$ Since the variables of interest are bounded to an interval, the least informative structure (i.e., maximizing Entropy) is a uniform distribution.
}

Algorithm The Basic MCMC Metropolis Algorithm: Given $x^{(i)}$,

1) Generate $y_{i} \sim q\left(y \mid x^{(i)}\right)$.

2) Take

$$
x^{(i+1)}= \begin{cases}y_{i} & \text { with probability }\left(\rho\left(x^{(i)}, y_{i}\right)\right) \\ x^{(i)} & \text { with probability }\left(1-\rho\left(x^{(i)}, y_{i}\right)\right)\end{cases}
$$

where $\rho(x, y)=\min \{(f(y) / f(x)), 1\}$.

\section{Sampling from the Posterior Density}

The problem we are dealing with now, is to obtain an MCMC sample $X^{(i)}=(r, \theta, v, \alpha)^{(i)}$ drawn from the joint posterior distribution $f(r, \theta, v, \alpha)=\pi[(r, \theta, v, \alpha) \mid$ $\left.\hat{\theta}_{1: N}\right]$. Firstly, a transition kernel is defined by

$$
\begin{aligned}
& q\left(\left(r^{\prime}, \theta^{\prime}, v^{\prime}, \alpha^{\prime}\right) \mid(r, \theta, v, \alpha)^{(i)}\right) \\
& \quad=q\left(r^{\prime} \mid r^{(i)}\right) \times q\left(\theta^{\prime} \mid \theta^{(i)}\right) \times q\left(v^{\prime} \mid v^{(i)}\right) \times q\left(\alpha^{\prime} \mid \alpha^{(i)}\right)
\end{aligned}
$$

where

$$
\begin{aligned}
& q\left(r^{\prime} \mid r^{(i)}\right) \sim U\left[r^{(i)}-\left(\frac{r_{\max }-r_{\min }}{\kappa_{1}}\right), r^{(i)}+\left(\frac{r_{\max }-r_{\min }}{\kappa_{1}}\right)\right] \\
& q\left(\theta^{\prime} \mid \theta^{(i)}\right) \sim U\left[\theta^{(i)}-\frac{10 \sigma}{\kappa_{2}}, \theta^{(i)}+\frac{10 \sigma}{\kappa_{2}}\right] \\
& q\left(v^{\prime} \mid v^{(i)}\right) \sim U\left[v^{(i)}-\left(\frac{v_{\max }-v_{\min }}{\kappa_{3}}\right), v^{(i)}+\left(\frac{v_{\max }-v_{\min }}{\kappa_{3}}\right)\right] \\
& q\left(\alpha^{\prime} \mid \alpha^{(i)}\right) \sim U\left[\alpha^{(i)}-\frac{2 \pi}{\kappa_{4}}, \alpha^{(i)}+\frac{2 \pi}{\kappa_{4}}\right] .
\end{aligned}
$$

Scalar parameters (see (12)) $\kappa_{1}, \kappa_{2}, \kappa_{3}$, and $\kappa_{4}$ (e.g. equal to 20) are introduced for algorithmic reasons, simply for avoiding that new values $\left(r^{\prime}, \theta^{\prime}, v^{\prime}, \alpha^{\prime}\right)$ be drawn out of the posterior distribution support and be rejected. Indeed, the above transition kernel satisfies the symmetry condition since we have

$-a \leq y-x \leq a \Leftrightarrow-a \leq x-y \leq a$,

so that

$$
q(x \mid y)=\frac{1}{2 a} \mathbf{1}_{[y-a, y+a]}(x)=\frac{1}{2 a} \mathbf{1}_{[x-a, x+a]}(y)=q(y \mid x) .
$$

Now, the next step is the computation of the (test) functional $\rho\left((r, \theta, v, \alpha)^{(i)},\left(r^{\prime}, \theta^{\prime}, v^{\prime}, \alpha^{\prime}\right)\right)$ which reduces to the computation of the ratio of the posterior densities:

$$
\begin{aligned}
& \frac{\pi\left[\left(r^{\prime}, \theta^{\prime}, v^{\prime}, \alpha^{\prime}\right) \mid \hat{\theta}_{1: N}\right]}{\pi\left[(r, \theta, v, \alpha)^{(i)} \mid \hat{\theta}_{1: N}\right]} \\
& =\frac{L_{\hat{\theta}_{1: N}}\left(r^{\prime}, \theta^{\prime}, v^{\prime}, \alpha^{\prime}\right) \times \pi\left(r^{\prime}, \theta^{\prime}, v^{\prime}, \alpha^{\prime}\right)}{L_{\hat{\theta}_{1: N}}(r, \theta, v, \alpha)^{(i)} \times \pi(r, \theta, v, \alpha)^{(i)}} \\
& =\frac{L_{\hat{\theta}_{1: N}}\left(r^{\prime}, \theta^{\prime}, v^{\prime}, \alpha^{\prime}\right)}{L_{\hat{\theta}_{1: N}}(r, \theta, v, \alpha)^{(t)}} \\
& \quad \times \mathbf{1}_{\left[r_{\min }, r_{\max }\right]}\left(r^{\prime}\right) \times \mathbf{1}_{\left[\theta_{\min }, \theta_{\max }\right]}\left(\theta^{\prime}\right) \times \mathbf{1}_{\left[v_{\min }, v_{\max }\right]}\left(v^{\prime}\right)
\end{aligned}
$$


itself requiring the calculation of the measurement likelihood. Assuming that the observations are normally distributed, i.e., $\hat{\theta}_{k}=\theta_{k}+\epsilon_{k}$ with $\epsilon_{k} \sim$ $N\left(0, \sigma^{2}\right)$, the elementary likelihood of the state vector $(r, \theta, v, \alpha)$ for the single bearing $\theta_{k}$ is given by

$$
L_{\hat{\theta}_{k}}(r, \theta, v, \alpha)=\frac{1}{\sigma \sqrt{2 \pi}} \exp \left[-\frac{1}{2} \frac{\left(\hat{\theta}_{k}-\theta_{k}[(r, \theta, v, \alpha)]\right)^{2}}{\sigma^{2}}\right]
$$

where $\theta_{k}[(r, \theta, v, \alpha)]$ is the exact bearing at the time period $\tau_{k}$ under the hypothesis that the target trajectory is defined by the state vector $(r, \theta, v, \alpha)$. Then, under the independence assumption, the ratio $(R)$ of the posterior densities reduces to

$$
\begin{aligned}
& \frac{\pi\left[\left(r^{\prime}, \theta^{\prime}, v^{\prime}, \alpha^{\prime}\right) \mid \hat{\theta}_{1: N}\right]}{\pi\left[(r, \theta, v, \alpha)^{(i)} \mid \hat{\theta}_{1: N}\right]} \\
& =\prod_{k=1}^{N}\left\{\frac{\exp \left[-\frac{1}{2 \sigma^{2}}\left(\hat{\theta}_{k}-\theta_{k}\left[\left(r^{\prime}, \theta^{\prime}, v^{\prime}, \alpha^{\prime}\right)\right]\right)^{2}\right]}{\exp \left[-\frac{1}{2 \sigma^{2}}\left(\hat{\theta}_{k}-\theta_{k}\left[(r, \theta, v, \alpha)^{(i)}\right]\right)^{2}\right]}\right\} \\
& \quad \times \mathbf{1}_{\left[r_{\min }, r_{\max }\right]}\left(r^{\prime}\right) \times \mathbf{1}_{\left[\theta_{\min }, \theta_{\max }\right]}\left(\theta^{\prime}\right) \times \mathbf{1}_{\left[v_{\min }, v_{\max }\right]}\left(v^{\prime}\right) .
\end{aligned}
$$

Now, we are in position to present the general form of the MCMC algorithm for simulating state vectors $(r, \theta, v, \alpha)^{(i)}$ distributed from the joint posterior density $\pi\left[(r, \theta, v, \alpha) \mid \hat{\theta}_{1: N}\right]$.

\section{AlgORITHM The BOT MCMC Algorithm: Define}

$(r, \theta, v, \alpha)^{(0)}=\left(\frac{r_{\min }+r_{\max }}{2}, \frac{\theta_{\min }+\theta_{\max }}{2}, \frac{v_{\min }+v_{\max }}{2}, 0\right)$

and set $i=0$ (choose an initial vector in Support $\left(\pi\left[(r, \theta, v, \alpha) \mid \hat{\theta}_{1: N}\right]\right)$.

Given $(r, \theta, v, \alpha)^{(i)}$

1) Generate $\left(r^{\prime}, \theta^{\prime}, v^{\prime}, \alpha^{\prime}\right)$ from:

$$
\begin{aligned}
& q\left(r^{\prime} \mid r^{(i)}\right) \sim U\left[r^{(i)}-\left(\frac{r_{\max }-r_{\min }}{\kappa_{1}}\right), r^{(i)}+\left(\frac{r_{\max }-r_{\min }}{\kappa_{1}}\right)\right] \\
& q\left(\theta^{\prime} \mid \theta^{(i)}\right) \sim U\left[\theta^{(i)}-\frac{10 \sigma}{\kappa_{2}}, \theta^{(i)}+\frac{10 \sigma}{\kappa_{2}}\right] \\
& q\left(v^{\prime} \mid v^{(i)}\right) \sim U\left[v^{(i)}-\left(\frac{v_{\max }-v_{\min }}{\kappa_{3}}\right), v^{(i)}+\left(\frac{v_{\max }-v_{\min }}{\kappa_{3}}\right)\right] \\
& q\left(\alpha^{\prime} \mid \alpha^{(i)}\right) \sim U\left[\alpha^{(i)}-\frac{2 \pi}{\kappa_{4}}, \alpha^{(i)}+\frac{2 \pi}{\kappa_{4}}\right] .
\end{aligned}
$$

\section{2) Choose}

$$
(\mathrm{r}, \theta, \mathrm{v}, \alpha)^{(\mathrm{i}+1)}= \begin{cases}\left(r^{\prime}, \theta^{\prime}, v^{\prime}, \alpha^{\prime}\right) & \text { with probability }(\rho) \\ (r, \theta, v, \alpha)^{(i)} & \text { with probability }(1-\rho)\end{cases}
$$

where $\rho=\min (R, 1)$ with $R$ the ratio of the posterior densities expressed by (16).

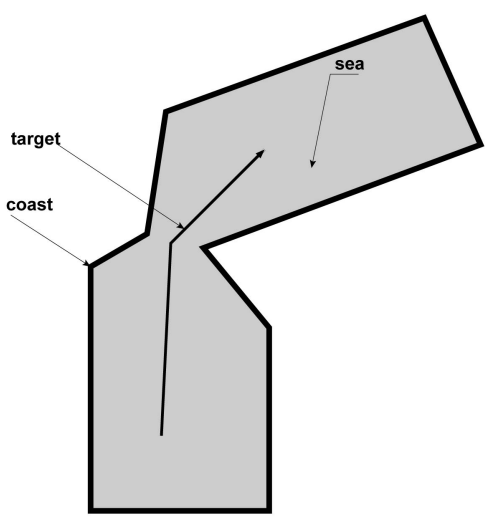

Fig. 7. Complex constraints on target trajectory.

\section{INCLUSION OF GENERAL CONSTRAINTS. THE MCMC HIT-AND-RUN ALGORITHM}

Actually, operational considerations frequently lead us to consider that the target trajectory lies in a given domain and that the target speed is bounded above and below. It is quite frequent that the constraint domain cannot be described directly only in terms of kinematic parameter intervals, thus implying complex prior density. ${ }^{11}$ This is illustrated by Fig. 7 .

For this context, the Metropolis-Hastings algorithm [18] cannot be used straightforwardly and we use instead the more versatile hit-and-run algorithm [7]. Hit-and-run algorithm originates from the same ideas than the Metropolis one; but departs with the transition kernel which is replaced by directly drawing on the constraint domain. Its original form has been proposed independently by Boneh and Golan [3] (1979) and Smith (1980) [38] for generating points uniformly distributed over bounded regions for solving optimization problems. Smith (1984) [39] proved the convergence of the algorithm, which has been extended by Belisle, Romeijn, and Smith [5] and Chen and Schmeiser [7].

\section{A. MCMC Hit-and-Run Sampling Algorithm}

Similarly to the Metropolis algorithm, the hit-and-run sampler is an MCMC method for generating random samples from an arbitrary continuous density $f$ over its support by drawing from a time-reversible Markov chain. The Markov chain transitions are defined by choosing a random direction and then moving to a new point whose likelihood depends on $f$ in that direction. The convergence is based on convergence in distribution of realizations to their asymptotic distribution $f$. The hit-and-run sampler, which generates a continuous-state Markov chain sample path $\left\{\mathbf{x}_{i}, i \geq 0\right\}$ from $S$, the support of $f$, is given below.

\footnotetext{
${ }^{11}$ Which means that it is unrealistic to incorporate these constrains, described by a set of equations, in a classical nonlinear optimization problem.
} 

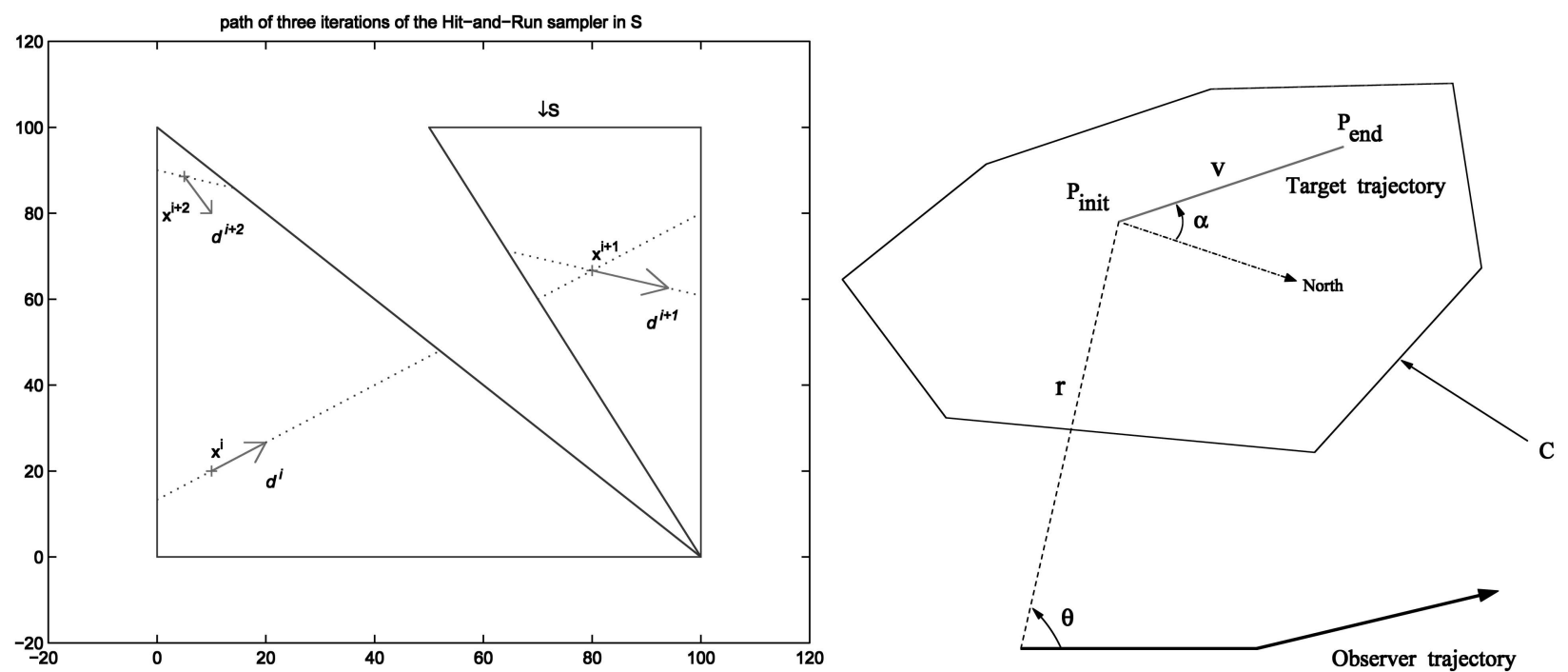

Fig. 8. Left: Hit-and-run algorithm. Right: rectilinear target trajectory under constraints.

\section{ALGORITHM Hit-and-Run Sampler:}

1) Choose a starting point $\mathbf{x}_{0} \in S$, and set $i=0$.

2) Generate a direction $\mathbf{d}_{i}$, from a distribution on the surface of the unit sphere.

3) Find the set $S_{i}\left(\mathbf{d}_{i}, \mathbf{x}_{i}\right) \triangleq\left\{\lambda \in \mathbb{R} \mid \mathbf{x}_{i}+\lambda \mathbf{d}_{i} \in S\right\}$.

4) Generate a signed distance: $\lambda_{i}$ from density $g_{i}\left(\lambda \mid \mathbf{d}_{i}, \mathbf{x}_{i}\right)$, where $\lambda_{i} \in S_{i}$.

5) Set $\mathbf{y}=\mathbf{x}_{i}+\lambda_{i} \mathbf{d}_{i}$

$$
\mathbf{x}_{i+1}=\left\{\begin{array}{ll}
\mathbf{y} & \text { with probability }\left(a_{i}\left(\mathbf{y} \mid \mathbf{x}_{i}\right)\right), \\
\mathbf{x}_{i} & \text { otherwise }
\end{array} .\right.
$$

6) Set $i+1$, and go to Step 2 .

Various choices exist for the distribution of $\mathbf{d}_{i}$, the densities $g_{i}$, and the probabilities $a_{i}$ [23]. It is not necessary for the density function $f$ or its support to be bounded [7]. In practice, there are various jump strategies (i.e., choices of $g_{i}\left(\lambda \mid \mathbf{d}_{i}, \mathbf{x}_{i}\right)$ and $\left.a_{i}\left(y \mid \mathbf{x}_{i}\right)\right)$ allowing the resulting Markov chain to have a probability transition kernel and to be time reversible with respect to $f$. As we consider a target moving in a bounded area, the following strategy is used:

1) $g_{i}\left(\lambda \mid \mathbf{d}_{i}, \mathbf{x}_{i}\right)=1 /\left(m\left[S_{i}\left(\mathbf{d}_{i}, \mathbf{x}_{i}\right)\right]\right)$ for $\lambda \in S_{i}\left(\mathbf{d}_{i}, \mathbf{x}_{i}\right)$ where $m$ is Lebesgue measure,

2)

$$
a_{i}\left(\mathbf{y} \mid \mathbf{x}_{i}\right)= \begin{cases}\frac{f(\mathbf{y})}{f\left(\mathbf{x}_{i}\right)+f(\mathbf{y})} & \text { (Barker's method) } \\ \min \left(1, \frac{f(\mathbf{y})}{f\left(\mathbf{x}_{i}\right)}\right) & \text { (Metropolis's method). }\end{cases}
$$

Directions $\mathbf{d}_{i} \in \mathbb{R}^{n}$ point on the surface of the unit sphere and are obtained by drawing $D_{i}^{j} \sim U[-1,1]$ for $j=1 \ldots n$ (where $U$ represents the uniform law) and by normalizing to 1 so that the $j$ th component of the $\mathbf{d}_{i}$ vector is $\mathbf{d}_{i}^{j}=D_{i}^{j} /\left[\sum_{k=1}^{n}\left(D_{i}^{k}\right)^{2}\right]^{1 / 2}$. We now examine how to apply the hit-and-run in the BOT TMA context.

\section{B. Rectilinear Target Trajectory}

Here, the problem we have to deal with is to draw samples $(r, \theta, v, \alpha)^{(i)}$ from the posterior density $\pi\left[(r, \theta, v, \alpha) \mid \hat{\theta}_{1: N}\right]$, considering both prior about target speed and feasibility of the target trajectory. Under the assumption of a rectilinear and uniform motion, the target trajectory (denoted $\mathcal{T}$ ) can be defined by its two extremal points $\left(P_{\text {init }}\right.$ and $\left.P_{\text {end }}\right)$, i.e.,

$$
\begin{aligned}
P_{\text {init }} & =\left(\begin{array}{l}
r_{x, \text { init }} \\
r_{y, \text { init }}
\end{array}\right) \in \mathbb{R}^{2} \\
P_{\text {end }} & =\left(\begin{array}{l}
r_{x, \text { end }} \\
r_{y, \text { end }}
\end{array}\right) \in \mathbb{R}^{2} \\
\mathcal{T} & =\left(\begin{array}{c}
P_{\text {init }} \\
P_{\text {end }}
\end{array}\right) .
\end{aligned}
$$

Let us denote $C$ the area where stand all the target trajectories without constraint on the maximal speed as represented in Fig. 8. Iterations of the hit-and-run algorithm are represented in this figure, for a complex domain $C$.

Now let $S \in \mathbb{R}^{2} \times \mathbb{R}^{2}$ be the subset of $C$ representing all the target trajectories satisfying both the constraint on the maximum target speed and the feasibility constraints. $S$ is then defined by the relation $(C$ convex):

$$
\mathcal{T}=\left(\begin{array}{l}
P_{\text {init }} \\
P_{\text {end }}
\end{array}\right) \in S \Longleftrightarrow\left\{\begin{array}{l}
P_{\text {init }} \in C \\
P_{\text {end }} \in C \\
\left\|P_{\text {end }}-P_{\text {init }}\right\| \leq L
\end{array}\right.
$$

where $L$ is the maximal length of a trajectory defined from the total measurement duration $\Delta t$ and the target speed upper bound $v_{\max }$; so that $L=v_{\max } \times \Delta t$. In this setup, the hit-and-run algorithm is applied to TMA by associating a point $x$ in $S$ with a feasible trajectory $\mathcal{T}$, and is detailed below. 


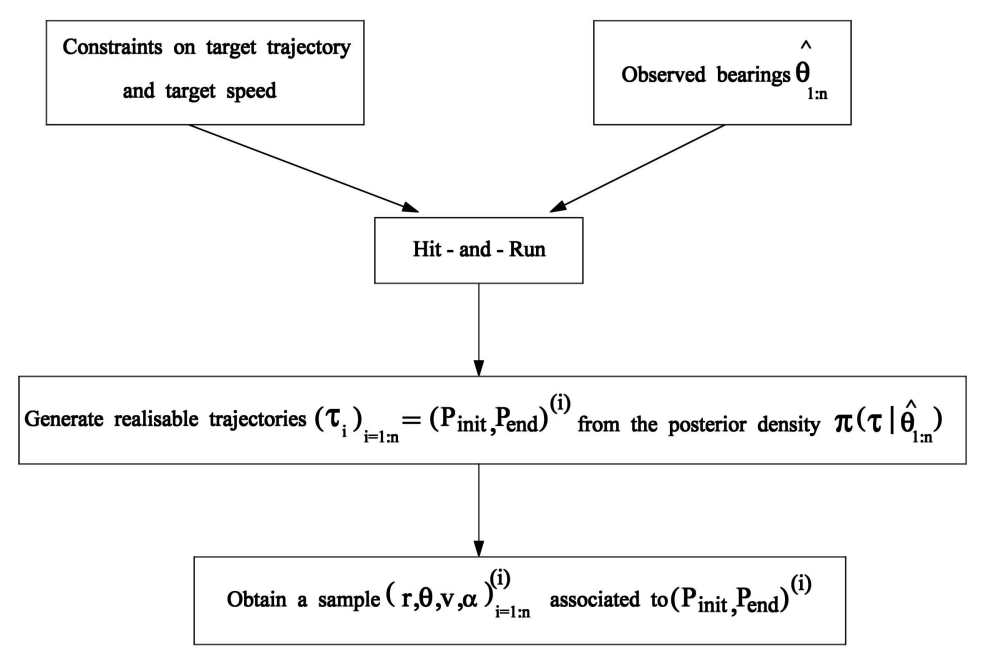

Fig. 9. Rectilinear target trajectory under constraints.

\section{AlgORITHM Hit-and-Run Sampler:}

1) Choose an initial arbitrary trajectory satisfying the constraints. Define $\mathcal{T}_{0}$ as:

$$
\mathcal{T}_{0}=\left(\begin{array}{c}
P_{\text {init }} \\
P_{\text {end }}
\end{array}\right)^{(0)} \in S .
$$

2) For $i=1, \ldots$, generate 4 uniform variables in $[-1,1]:\left\{D_{1}, D_{2}, D_{3}, D_{4}\right\}$ that represent the changes for the points $P_{\text {init }}$ and $P_{\mathrm{end}}$ and define the normalized $\mathbf{d}_{i}$ vector $\left(\left\|\mathbf{d}_{i}\right\|=1\right)$ by

$$
\mathbf{d}_{i}=\left(\begin{array}{c}
d_{1}^{(i)} \\
d_{2}^{(i)} \\
d_{3}^{(i)} \\
d_{4}^{(i)}
\end{array}\right) .
$$

3) Determine the $\lambda$ set: $S_{i}\left(\mathbf{d}_{i}, \mathcal{T}_{i}\right) \triangleq\left\{\lambda \in \mathbb{R} \mid \mathcal{T}_{i}+\right.$ $\left.\lambda \mathbf{d}_{i} \in S\right\}$. Then, the constraints on $\lambda$ stand as follows:

$$
\mathcal{T}_{i}+\lambda \mathbf{d}_{i}=\left(\begin{array}{c}
r_{x, \text { init }}^{(i)}+\lambda d_{1}^{(i)} \\
r_{y, \text { init }}^{(i)}+\lambda d_{2}^{(i)} \\
r_{x, \text { end }}^{(i)}+\lambda d_{3}^{(i)} \\
r_{y, \text { end }}^{(i)}+\lambda d_{4}^{(i)}
\end{array}\right) \in S
$$

i.e.,

$$
\left\{\begin{array}{l}
\left(\begin{array}{c}
r_{x, \text { init }} \\
r_{y, \text { init }}
\end{array}\right)^{(i)}+\lambda\left(\begin{array}{c}
d_{1}^{(i)} \\
d_{2}^{(i)}
\end{array}\right) \in C \quad\left(\begin{array}{c}
r_{x, \text { end }} \\
r_{y, \text { end }}
\end{array}\right)^{(i)}+\lambda\left(\begin{array}{c}
d_{3}^{(i)} \\
d_{4}^{(i)}
\end{array}\right) \in C, \\
\left\|\left(\begin{array}{l}
r_{x, \text { end }}-r_{x, \text { nit }} \\
r_{y, \text { end }}-r_{y, \text { init }}
\end{array}\right)^{(i)}+\lambda\left(\begin{array}{c}
d_{3}^{(i)}-d_{1}^{(i)} \\
d_{4}^{(i)}-d_{2}^{(i)}
\end{array}\right)\right\| \leq L .
\end{array}\right.
$$

4) Draw a signed distance $\lambda_{i}$ from the density:

$$
g_{i}\left(\lambda \mid \mathbf{d}_{i}, \mathcal{T}_{i}\right)=\frac{1}{m\left(S_{i}\left(\mathbf{d}_{i}, \mathcal{T}_{i}\right)\right)} \mathbf{1}_{S_{i}\left(\mathbf{d}_{i}, \mathcal{T}_{i}\right)}(\lambda)
$$

where $m$ is the (Lebesgue) measure of the segment $S_{i}\left(\mathbf{d}_{i}, \mathcal{T}_{i}\right)$.
5) Define the $\Phi$ vector by $\Phi=\mathcal{T}_{i}+\lambda_{i} \cdot \mathbf{d}_{i}$ and define $\mathcal{T}_{i+1}$ by:

$$
\mathcal{T}_{i+1}= \begin{cases}\Phi & \text { with probability } \min \left(1, \frac{f(\Phi)}{f\left(\mathcal{T}_{i}\right)}\right) \\ \mathcal{T}_{i} & \text { otherwise, }\end{cases}
$$

where $f(\Phi)$ and $f\left(\mathcal{T}_{i}\right)$ are the posterior distributions of the trajectories $\Phi$ and $\mathcal{T}_{i}$ respectively.

In fact, the ratio of the posterior densities $f(\Phi) / f\left(\mathcal{T}_{i}\right)$ reduces to the likelihood ratio since the prior information is already naturally taken into account in the generation of feasible trajectories by the algorithm, thus:

$$
\frac{f(\Phi)}{f\left(\mathcal{T}_{i}\right)}=\frac{L_{\hat{\theta}_{1: N}}(\Phi)}{L_{\hat{\theta}_{1: N}}\left(\mathcal{T}_{i}\right)}
$$

where $L_{\hat{\theta}_{1: N}}(\Phi)$ and $L_{\hat{\theta}_{1: N}}\left(\mathcal{T}_{i}\right)$ are likelihoods of the trajectories $\Phi$ and $\mathcal{T}_{i}$ respectively. For a given trajectory $\Phi$, the likelihood functional is classically calculated via (see 15):

$$
L_{\hat{\theta}_{k}}(\Phi)=\frac{1}{\sigma \sqrt{2 \pi}} \exp \left[-\frac{1}{2} \frac{\left(\hat{\theta}_{k}-\theta_{k}[\Phi]\right)^{2}}{\sigma^{2}}\right]
$$

and (independence assumption):

$$
L_{\hat{\theta}_{1: N}}(\Phi)=\prod_{k=1}^{N} L_{\hat{\theta}_{k}}(\Phi)
$$

where $\theta_{k}[\Phi]$ is the exact bearing at the time period $\tau_{k}$ related to the trajectory $\Phi$. The functional $L_{\hat{\theta}_{1: N}}\left(\mathcal{T}_{i}\right)$ is identically defined. The following proposition, though elementary, is especially relevant for applying the hit-and-run algorithm (see Appendix C, for a proof).

Proposition 3 Assume that the constraint domain C is convex, then the set of feasible trajectories $S$ is also convex. 
Therefore, at step 3 of the above algorithm, $S_{i}\left(\mathbf{d}_{i}, \mathcal{T}_{i}\right)$ is simply a real interval $\left[\lambda_{\min }, \lambda_{\max }\right]$ satisfying the following conditions: $\mathcal{T}_{i}+\lambda_{\min } \cdot \mathbf{d}_{i} \in \operatorname{Fr}(S)$ or $\mathcal{T}_{i}+$ $\lambda_{\text {max }} \cdot \mathbf{d}_{i} \in \operatorname{Fr}(S)$. The whole algorithm is summarized in Fig. 9.

The above method deals with a target located in a known area and moving in a straight line with constant speed. We now see how this method can handle scenarios of increasing complexity.

\section{Hit-and-Run Algorithm and the Leg-by-Leg Target Trajectory Model}

We are now dealing with the estimation of leg-by-leg target trajectory parameters. The target trajectory is supposed made of two legs. The constraint expresses that the first leg and the second leg of the target trajectory stand in given areas. A new state vector has to be considered. It seems natural to consider the state vector made of the three points that characterize the leg-by-leg trajectory. In this setting, the target trajectory $\mathcal{T}$ can be defined by three "characteristic" points $\left(P_{\text {init }}, P_{\text {mid }}\right.$, and $\left.P_{\text {end }}\right)$, so that

$$
\begin{gathered}
\mathcal{T} \stackrel{\text { def }}{=}\left(P_{\text {init }}, P_{\text {mid }}, P_{\text {end }}\right)^{T}, \quad P_{\text {init }}=\left(\begin{array}{c}
r_{x, \text { init }} \\
r_{y, \text { init }}
\end{array}\right) \in \mathbb{R}^{2} \\
P_{\text {mid }}=\left(\begin{array}{c}
r_{x, \text { mid }} \\
r_{y, \text { mid }}
\end{array}\right) \in \mathbb{R}^{2}, \quad P_{\text {end }}=\left(\begin{array}{c}
r_{x, \text { end }} \\
r_{y, \text { end }}
\end{array}\right) \in \mathbb{R}^{2} .
\end{gathered}
$$

Denote $C_{1}$ the area where the first target trajectory leg stands: $\left[P_{\text {init }}, P_{\text {mid }}\right]$ and $C_{2}$ the area for the second leg: $\left[P_{\text {mid }}, P_{\text {end }}\right]$. Moreover, it is assumed that $P_{\text {mid }}$ stay in $C_{1} \cap C_{2}$. Moreover, let us denote $C=C_{1} \cup$ $C_{2}$ the area where all the target trajectories stand (without constraint on the maximal speed) and let $S \in \mathbb{R}^{2} \times \mathbb{R}^{2} \times \mathbb{R}^{2}$ be the $C$ subset representing all the target trajectories satisfying both the constraint on the maximum target speed and the constraint seen before on the leg-by-leg target trajectory. $S$ is defined by the relation:

$$
\mathcal{T}=\left(\begin{array}{c}
P_{\text {init }} \\
P_{\text {mid }} \\
P_{\text {end }}
\end{array}\right) \in S \Longleftrightarrow\left\{\begin{array}{l}
P_{\text {init }} \in C_{1} \\
P_{\text {mid }} \in C_{1} \cap C_{2} \\
P_{\text {end }} \in C_{2} \\
\left\|P_{\text {mid }}-P_{\text {init }}\right\|+\left\|P_{\text {end }}-P_{\text {mid }}\right\| \leq L
\end{array}\right.
$$

where $L$ is the maximal length of a trajectory defined from the total measurement duration $\Delta t$ and the maximal supposed target speed $v_{\max }$; so that $L=$ $v_{\max } \times \Delta t$. In this setup, the hit-and-run algorithm is applied to TMA by associating a point $x$ in $S$ to a feasible leg-by-leg trajectory $\mathcal{T}$ in $S$, thus taking the following form.
1) Choose an initial arbitrary trajectory satisfying the constraints. Define $\mathcal{T}_{0}$ as

$$
\mathcal{T}_{0}=\left(\begin{array}{c}
P_{\text {init }} \\
P_{\text {mid }} \\
P_{\text {end }}
\end{array}\right)^{(0)} \in S
$$

and set $i=0$.

2) Generate six (6) uniform variables in $[-1,1]$ : $\left\{D_{1}, D_{2}, D_{3}, D_{4}, D_{5}, D_{6}\right\}$ that represent the evolutions of directions of the points $P_{\text {init }}, P_{\text {mid }}$, and $P_{\text {end }}$ and define the normalized $\mathbf{d}_{i}$ vector $\left(\left\|\mathbf{d}_{i}\right\|=1\right)$ by $\mathbf{d}_{i}^{T}=$ $\left(d_{1}^{(i)}, d_{2}^{(i)}, d_{3}^{(i)}, d_{4}^{(i)}, d_{5}^{(i)}, d_{6}^{(i)}\right)$.

3) Determine the $\lambda$ set: $S_{i}\left(\mathbf{d}_{i}, \mathcal{T}_{i}\right) \stackrel{\text { def }}{=}\left\{\lambda \in \mathbb{R} \mid \mathcal{T}_{i}+\lambda\right.$. $\left.\mathbf{d}_{i} \in S\right\}$. Then the constraints on $\lambda$ stand as follows:

$$
\begin{aligned}
& \mathcal{T}_{i}+\lambda \mathbf{d}_{i} \in S \\
& \left\|\left(\begin{array}{c}
r_{x, \text { mid }}^{(i)}-r_{x, \text { init }}^{(i)} \\
r_{y, \text { mid }}^{(i)}-r_{y, \text { init }}^{(i)}
\end{array}\right)+\lambda\left(\begin{array}{c}
d_{3}^{(i)}-d_{1}^{(i)} \\
d_{4}^{(i)}-d_{2}^{(i)}
\end{array}\right)\right\| \\
& \quad+\left\|\left(\begin{array}{c}
r_{x, \text { end }}^{(i)}-r_{x, \text { mid }}^{(i)} \\
r_{y, \text { end }}^{(i)}-r_{y, \text { mid }}^{(i)}
\end{array}\right)+\lambda\left(\begin{array}{c}
d_{5}^{(i)}-d_{3}^{(i)} \\
d_{6}^{(i)}-d_{4}^{(i)}
\end{array}\right)\right\| \leq L \\
& \text { where } \quad \mathbf{d}_{i}=\left(d_{1}^{(i)}, d_{2}^{(i)}, d_{3}^{(i)}, d_{4}^{(i)}, d_{5}^{(i)}, d_{6}^{(i)}\right)^{T} .
\end{aligned}
$$

4) Generate a signed distance $\lambda_{i}$ from the density:

$$
g_{i}\left(\lambda \mid \mathbf{d}_{i}, \mathcal{T}_{i}\right)=\frac{1}{m\left(S_{i}\left(\mathbf{d}_{i}, \mathcal{T}_{i}\right)\right)} \mathbf{1}_{S_{i}\left(\mathbf{d}_{i}, \mathcal{T}_{i}\right)}(\lambda)
$$

where $m$ is the (Lebesgue) measure of the segment $S_{i}\left(\mathbf{d}_{i}, \mathcal{T}_{i}\right)$.

5) Define the $\Phi$ vector by $\Phi=\mathcal{T}_{i}+\lambda_{i} \cdot \mathbf{d}_{i}$ and define $\mathcal{T}_{i+1}$ by

$\mathcal{T}_{i+1}=$
$\begin{cases}\Phi & \text { with probability } \min \left(1, \frac{f(\Phi)}{f\left(\mathcal{T}_{i}\right)}\right), \frac{f(\Phi)}{f\left(\mathcal{T}_{i}\right)}=\frac{L_{\hat{\theta}_{1: N}}(\Phi)}{L_{\hat{\theta}_{1: N}}\left(\mathcal{T}_{i}\right)} . \\ \mathcal{T}_{i} & \text { otherwise }\end{cases}$

Finally, considering the convexity issue, the following proposition holds true.

Proposition 4 Consider now that the target trajectory $\mathcal{T}$ is made of two legs $\mathcal{T}_{1}$ and $\mathcal{T}_{2}$, both lying in a convex set $C$, then the following result holds:

If $C$ is convex, then $S$ is convex.

The proof of this property is omitted since it is similar to that of Proposition 3 given in Appendix C. Now, a fundamental concern is the determination of confidence interval for the estimates we have obtained. To that aim, we exploit the MCMC procedures within the HPD-interval framework to determine confidence interval for range estimation. 


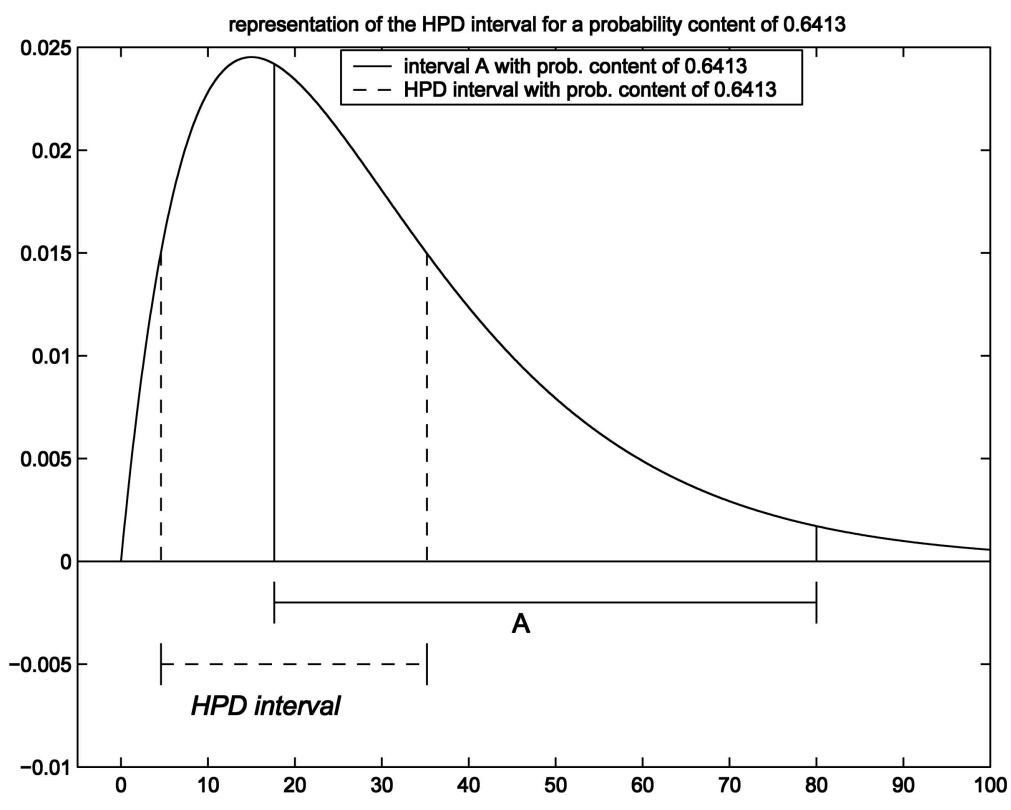

Fig. 10. Representation of HPD interval.

\section{HIGHEST PROBABILITY DENSITY INTERVALS}

Practically, it is of fundamental importance to have an estimation of confidence intervals for high level information processing: e.g., track-to-track association, sensor management, situation assessment, etc. We see now that MCMC-based methods give us estimates of these confidence intervals, as a by-product.

\section{A. HPD Intervals Method}

The aim of this paragraph is to present the calculation of the HPD intervals method and their connection with MCMC methods. Consider a Bayesian posterior density having the form:

$$
\pi(\lambda, \varphi \mid \mathcal{D})=\frac{L_{\mathcal{D}}(\lambda, \varphi) \times \pi(\lambda, \varphi)}{c(\mathcal{D})}
$$

where $\mathcal{D}$ denotes data, $\lambda$ is one-dimensional, $\varphi$ may be a multidimensional vector of parameters other than $\lambda$. The functional $L_{\mathcal{D}}(\lambda, \varphi)$ is a likelihood functional, $\pi(\lambda, \varphi)$ is a prior and $c(\mathcal{D})$ is a normalization constant. An HPD interval has two main properties:

1) the density for every point inside the interval is greater than that for every point outside the interval,

2) for a given probability content, say $1-\alpha$, the interval is of the shortest length.

Especially, it is when the marginal distribution is not symmetric (see Fig. 3), that an HPD interval is mostly desirable. Fig. 10 illustrates these properties. It presents a density function, an interval having a probability content of 0.6413 , and the HPD interval for this given probability. Tanner [40] provides a Monte Carlo algorithm to calculate the content and boundary of the HPD region. However,
Tanner's algorithm requires evaluating the marginal posterior densities analytically or numerically, which renders its implementation rather complicated and computationally intensive. To overcome such difficulties, Chen, Shao, and Ibrahim [9] developed a simple Monte Carlo method for estimating HPD intervals. This approach requires only an MCMC sample generated from the marginal posterior distribution of the parameter of interest and propose the following procedure [8] for calculating an HPD interval for $\lambda$ :

\section{Algorithm Chen-Shao HPD Estimation Algorithm}

1) Obtain an MCMC sample $\left\{\lambda_{i}\right\}_{i=1: n}$ from $\pi(\lambda \mid D)$.

2) Sort $\left\{\lambda_{i}\right\}_{i=1: n}$ to obtain the ordered values:

$$
\lambda_{(1)} \leq \lambda_{(2)} \leq \cdots \leq \lambda_{(n)} .
$$

3) Compute the $100(1-\alpha) \%$ credible intervals:

$$
R_{j}(n)=\left(\lambda_{(j)}, \lambda_{(j+[(1-\alpha) n])}\right)
$$

for $j=1: n-[(1-\alpha) n]$ (where $[\cdot]$ refers to the entire part).

4) The 100(1- $\alpha) \%$ HPD interval is the one, denoted by $R_{j^{*}}(n)$, with the smallest interval width among all credible intervals.

The main interest of this procedure is that it does not require a closed form of the marginal density. The following proposition, given by Gelfand and Smith [13] (1990), is particularly useful for our TMA problem.

PROPOSITION 5 If $\left\{\left(\lambda_{i}, \varphi_{i}\right)\right\}_{i=1: n}$ is an MCMC sample from the joint posterior distribution $\pi\left(\lambda, \varphi \mid \hat{\theta}_{1: N}\right)$ then $\left\{\lambda_{i}\right\}_{i=1: n}$ is an MCMC sample from the marginal posterior distribution $\pi\left(\lambda \mid \hat{\theta}_{1: N}\right)$. 
Let us consider now the application of this property to our context.

\section{B. Rectilinear Uniform Target Motion Case}

Whether the first or second type of constraints is considered, our aim is to provide a confidence interval for the initial range $r$. Thus, it remains to apply the HPD method for the range $r$, i.e., obtain an MCMC sample from the marginal posterior distribution of the parameter of interest, i.e., $\pi\left(r \mid \hat{\theta}_{1: N}\right)$. In Sections IIC and IVB, a sample $\left\{(r, \theta, v, \alpha)^{(i)}\right\}_{i=1: n}$ from the posterior density $\pi\left[(r, \theta, v, \alpha) \mid \hat{\theta}_{1: N}\right]$ of the complete state vector was obtained by using a MCMC technique according to the type of constraints considered.

Proposition 5 provides justification for MCMC sampling from the marginal posterior distribution by taking the suitable marginal component of the MCMC sample from the posterior distribution of the complete state vector. So, for determining a $100(1-\alpha) \%$ HPD interval for the range $r$ the following procedure is used.

1) Obtain an MCMC sample $\left\{(r, \theta, v, \alpha)^{(i)}\right\}_{i=1: n}$ from $\pi\left[(r, \theta, v, \alpha) \mid \hat{\theta}_{1: N}\right]$ as seen in Sections IIC and IVB according to the type of constraints considered.

2) Obtain an MCMC sample $\left\{r_{i}\right\}_{i=1: n}$ from $\pi\left(r \mid \hat{\theta}_{1: N}\right)$ by taking the first component of the precedent state vector.

3) Sort $\left\{r_{i}\right\}_{i=1: n}$ to obtain the ordered values:

$$
r_{(1)} \leq r_{(2)} \leq \cdots \leq r_{(n)} .
$$

4) Compute the $100(1-\alpha) \%$ credible intervals:

$$
R_{j}(n)=\left(r_{(j)}, r_{(j+[(1-\alpha) n])}\right)
$$

for $j=1: n-[(1-\alpha) n]$ (where [·] refers to the entire part).

5) The 100(1- $1-\alpha)$ HPD interval is the one, denoted by $R_{j^{*}}(n)$, with the smallest interval width among all credible intervals.

Considering the simplicity of these algorithms, the computational load of an iteration is very modest. However, the number of iterations may be relatively important, especially for obtaining reliable HPD estimates. A good test for stopping iterations is the stabilization of HPD estimates.

\section{RESULTS}

Roughly, this section is divided into two parts, whose common features are: the observer is nonmaneuvering, ${ }^{12}$ constraints are utilized for range estimation. The first one is devoted to the case where the target is nonmaneuvering, and in the second case the target performs a dog-leg maneuver. For

\footnotetext{
${ }^{12}$ More precisely, we allow to consider only very gentle observer maneuver so as to avoid strict observability problems.
}

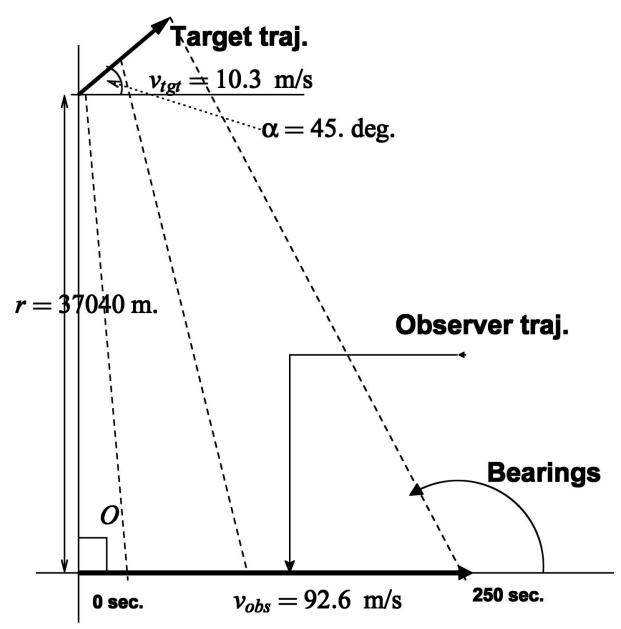

Fig. 11. Scenario parameters. Target parameters: $r=37040 \mathrm{~m}$; $v_{\mathrm{tgt}} \approx 10.3 \mathrm{~m} / \mathrm{s} ; \theta=90 \mathrm{deg} ; \alpha=45 \mathrm{deg}$.

both cases, it is target range estimation which is our principal concern.

\section{A. Nonmaneuvering Target}

Here, we restrict to BOT TMA for a nonmaneuvering target (rectilinear and uniform motion). The general scenario is depicted in Fig. 11.

1) Constraint on Target Speed: Priors Defined as Intervals: The first type of constraints we consider is target speed constraints (see Section III). Priors are defined by intervals for the kinematics parameters $\{r, \theta, v, \alpha\}$. The true target state vector is defined as $r=37,040 \mathrm{~m}, v \approx 10.3 \mathrm{~m} / \mathrm{s}$ (20 knots), $\theta=90 \mathrm{deg}$, $\alpha=45 \mathrm{deg}$, (at time 0 ). The observer speed is $92.6 \mathrm{~m} / \mathrm{s}$ (180 knots), and its turn angle (maneuver) is as less than $10^{-4} \mathrm{deg}$, so that (observer) motion can be considered rectilinear and uniform. Fifty (50) bearing measurements are taken with an interval of $5 \mathrm{~s}$ between each measurement. The measurement error is modelled by a zero-mean Gaussian noise, with a 1 deg standard deviation. Prior constraints stand as follows: $r \in[500,200,000] \mathrm{m}, \theta \in\left[\hat{\theta}_{1}-5 \sigma, \hat{\theta}_{1}+5 \sigma\right]$, $v \in[7.716,12.86](\mathrm{m} / \mathrm{s})$ and $\alpha \in[-\pi, \pi]$. It is clear that only speed constraints are really informative. The Metropolis algorithm generates a sample vector of dimension 100,000.

We present in Fig. 12 (left) the evolution of the MCMC sample for range generated from the marginal posterior density, taking into account the above constraints. The true range $(37,040 \mathrm{~m})$ is drawn as a solid line. It can be seen that the range estimates are (only) slightly greater than the true value. Considering that it is unobservable (without target speed constraints) this is a remarkable result.

Fig. 12 (right) illustrates the HPD interval width versus probability of content. Actually, it is the complement of this probability of content which is plotted on the $x$-axis (i.e., $1-P$ (prob. of content)). 

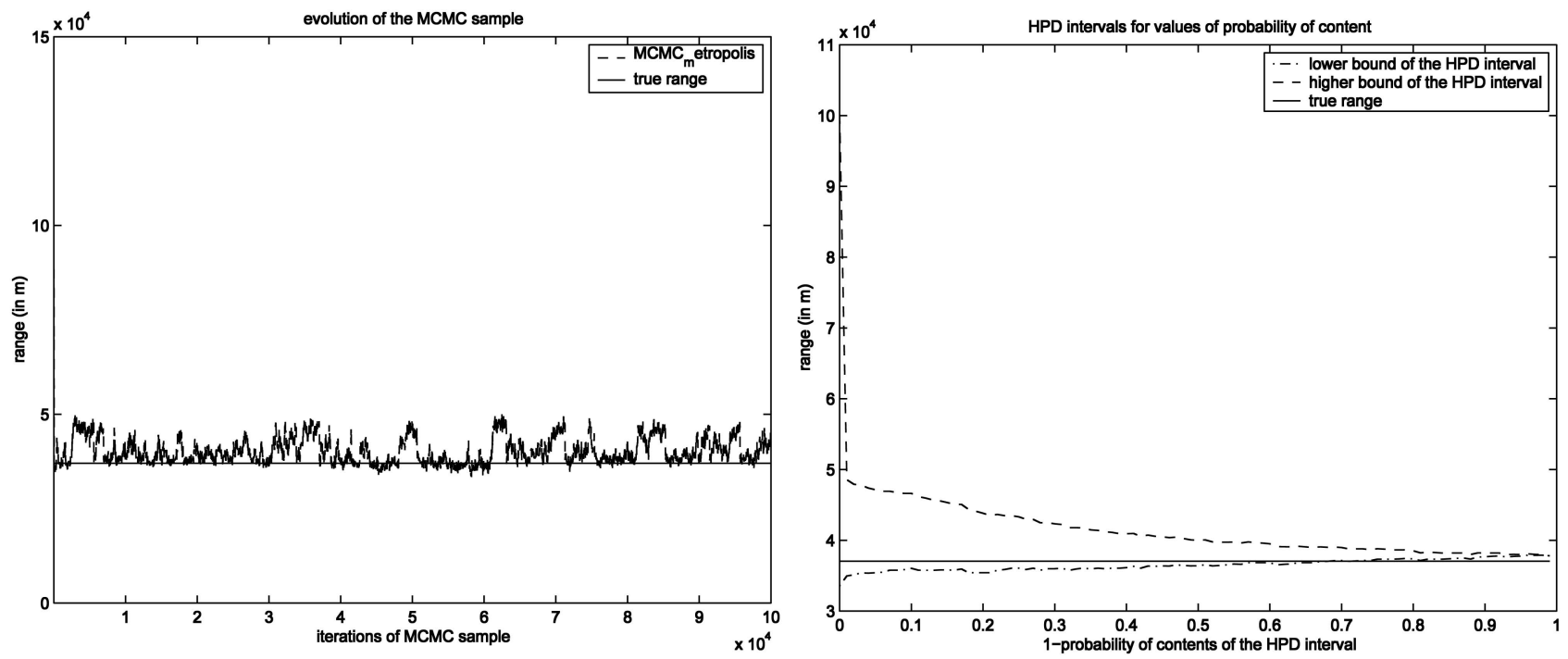

Fig. 12. Left: MCMC samples versus time. Right: HPD interval width versus probability of content.
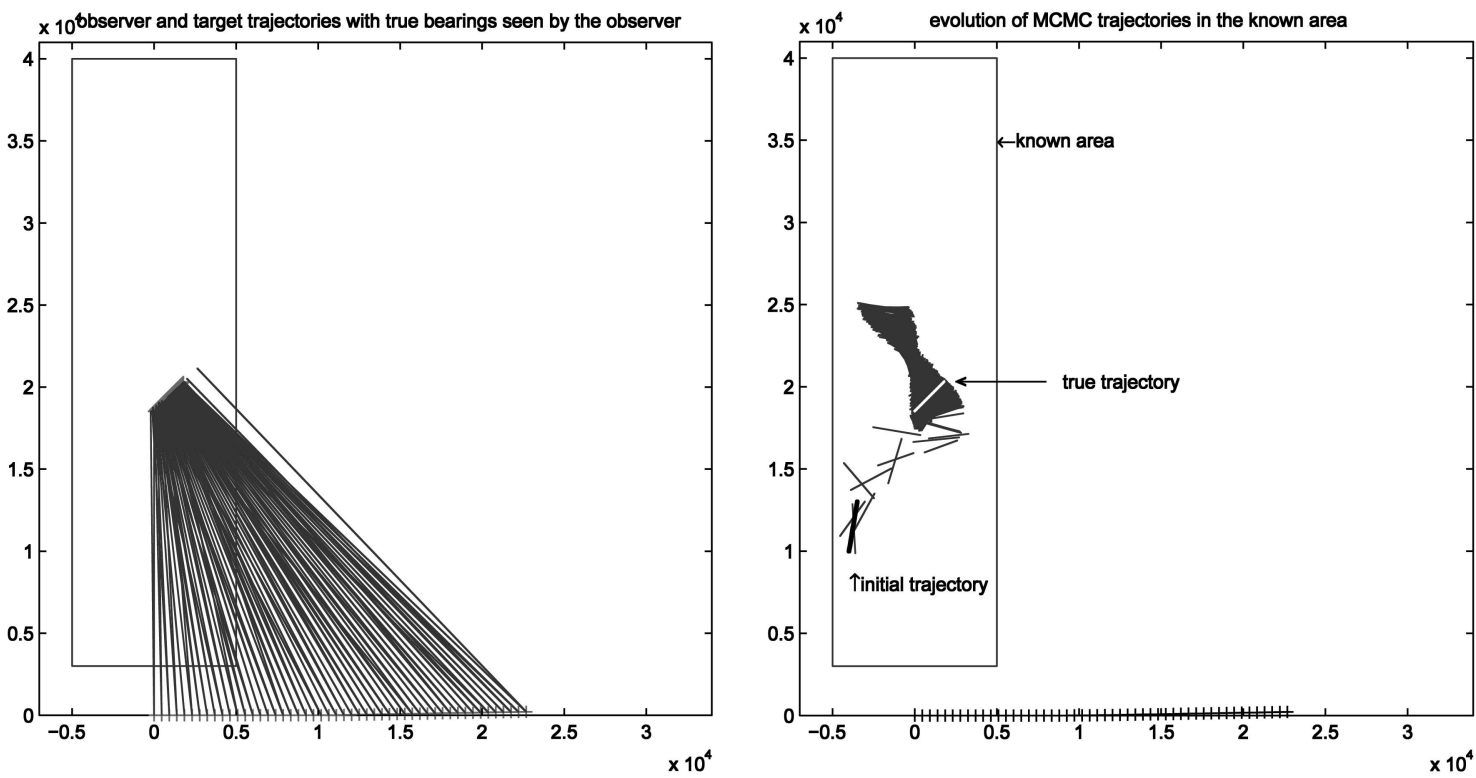

Fig. 13. Left: observations (sequence of estimated bearings). Right: 500 iterations of MCMC hit-and-run.

So, for abscissa 0, the HPD interval is $100 \%$ of the MCMC values, since this value gives the minimum and maximum range of the MCMC sampling. For every value of probability of content, the lower bound (respectively higher bound) of the HPD interval is represented by the dash-dot line (respectively the dashed line). The true value of range falls outside the HPD interval only when the probability of content is as low as 0.3. Even for this value, the HPD interval is close to the true value, while the interval width is as small as $2.5 \mathrm{~km}$. Moreover, for greater values of the probability of content, we can see that the lower bound is very close to the true range value and that the interval width remains very reasonable, which is a very satisfactory result. The benefit of including speed constraints is clear.
2) Constraints on Target Speed and Target Trajectory: The second result concerns the implementation of the hit-and-run algorithm using constraints about target trajectories (trajectory domain and target speed) (see Section IVB). The scenario is almost the same than previously, apart from the fact that the target range which is now $18,520 \mathrm{~m}$. In this example, the trajectory constraints are simple: all the target trajectories are supposed to lie inside a rectangle of length $37,000 \mathrm{~m}$ and width $10,000 \mathrm{~m}$, as depicted by the rectangle in Fig. 13, while it is assumed that target speed is bounded above by $v_{\max }=30$ knots. The noisy bearings measured by the observer are also represented in Fig. 13 (left). In Fig. 13 (right), we present 30,000 iterations of the MCMC hit-and-run, associated with this 

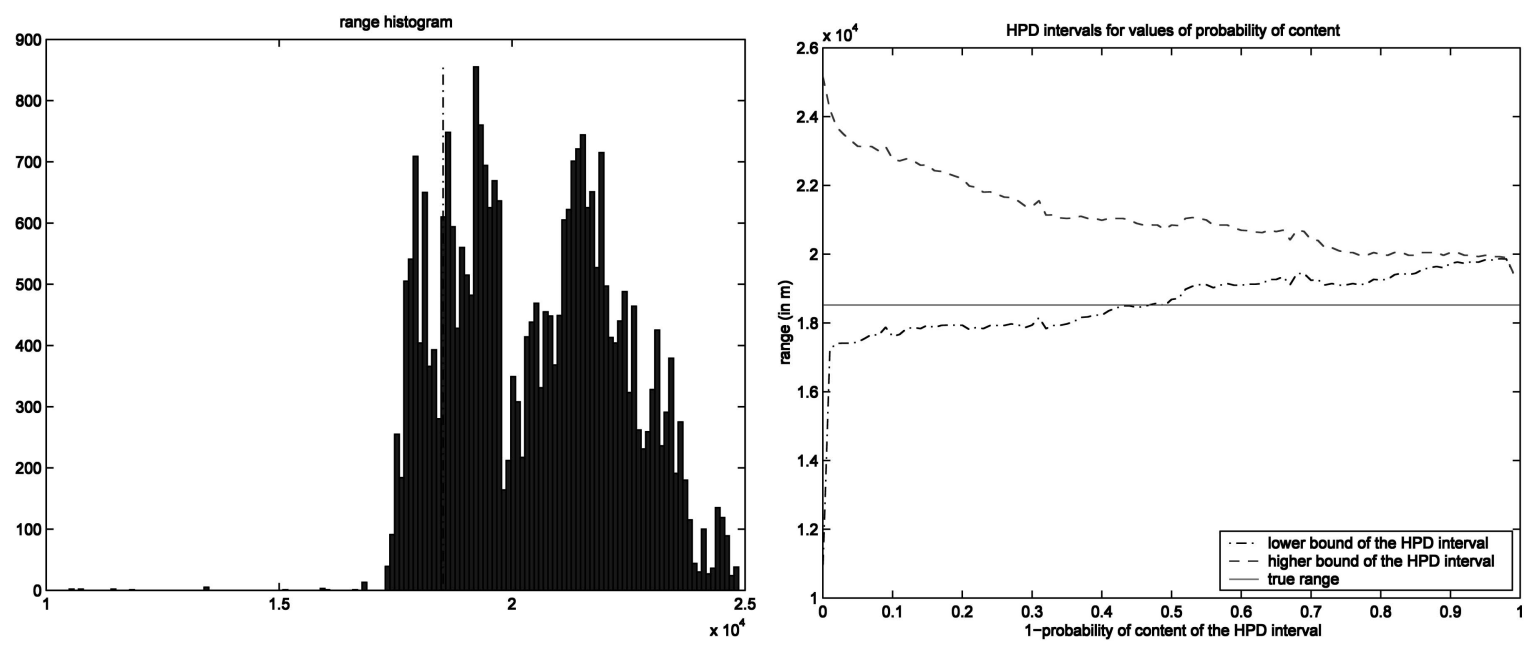

Fig. 14. Left: MCMC samples versus time. Right: HPD interval width versus probability of content.
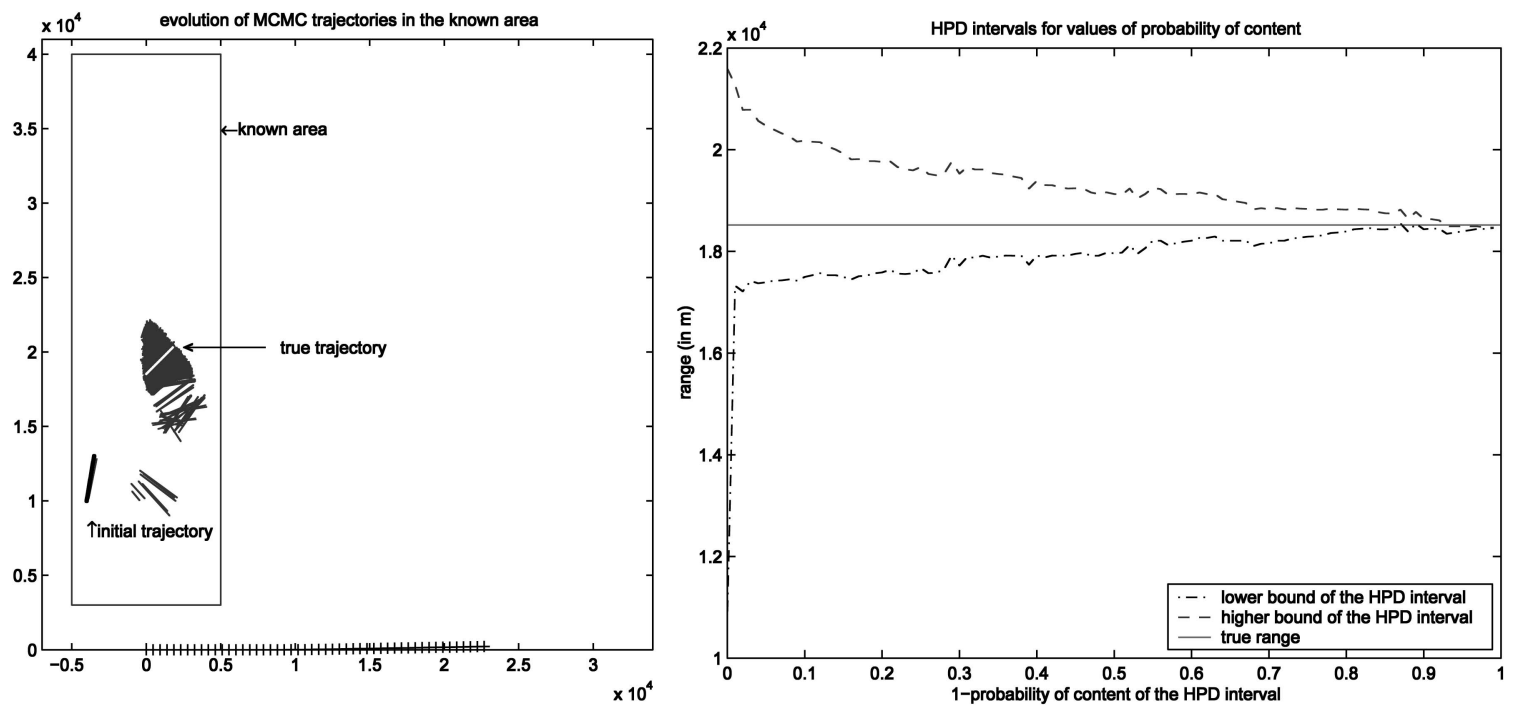

Fig. 15. Left: MCMC samples versus time. Right: HPD interval width versus probability of content.

sequence of observations and these constraints. Considering Fig. 13, we notice that the hit-and-run algorithm is rapidly "converging" toward a set of feasible target trajectories, roughly representing an "hourglass" (see Fig. 13 right). Thus, the hit-and-run algorithm provides an exploration of the uncertainty domain about target trajectory (see Proposition 2 and Appendix B). The choice of the initial trajectory (the segment down left) is unimportant provided that it satisfies the conditions 18 .

In order to evaluate the accuracy of HPD intervals calculated by this way, 30,000 samples (trajectories) are drawn and represented by the histogram in Fig. 14 (left). This histogram clearly exhibits two peaks: one is close to the true range value (dashed line), while the other is corresponding to ghost trajectories (see Fig. 5 for a geometric interpretation). In consequence, HPD intervals are also "pulled" by these ghost trajectories, as seen in Fig. 14 (right) which presents the evolution of the HPD intervals obtained via the hit-and-run algorithm. This event is also quite evident by considering the "hourglass" drawn by the feasible trajectories in Fig. 13 (right).

Then it is meaningful to consider an additional condition on the feasible trajectories directions so as to eliminate ghosting. This is the object of Fig. 15 (left) for which trajectory sampling is restricted to the true direction (i.e., left to right). MCMC estimation is clearly improved by deghosting. More generally, ghosting is also resulting from unobservability (see Appendix B) and deghosting is a fundamental concern for all these scenarios. Actually, it may be shown that even a very limited observer maneuver may be sufficient for ensuring good performance deghosting thanks to the calculation of appropriate likelihood ratio tests and reliable confidence intervals via HPD.

\section{B. Maneuvering Target}

The last part of this section deals with the leg-by-leg target trajectory case (see Section IVC). 

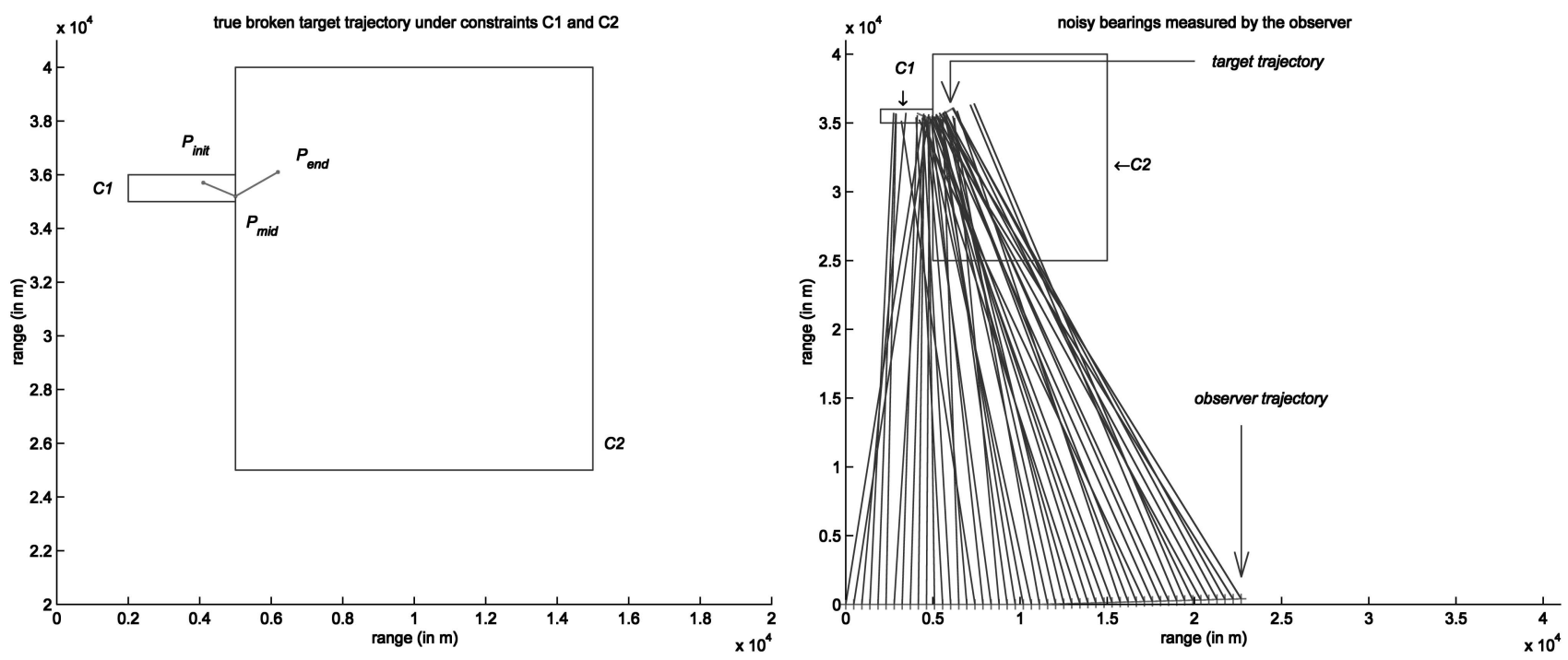

Fig. 16. Left: true target trajectory under set constraints. Right: noisy observed bearings.
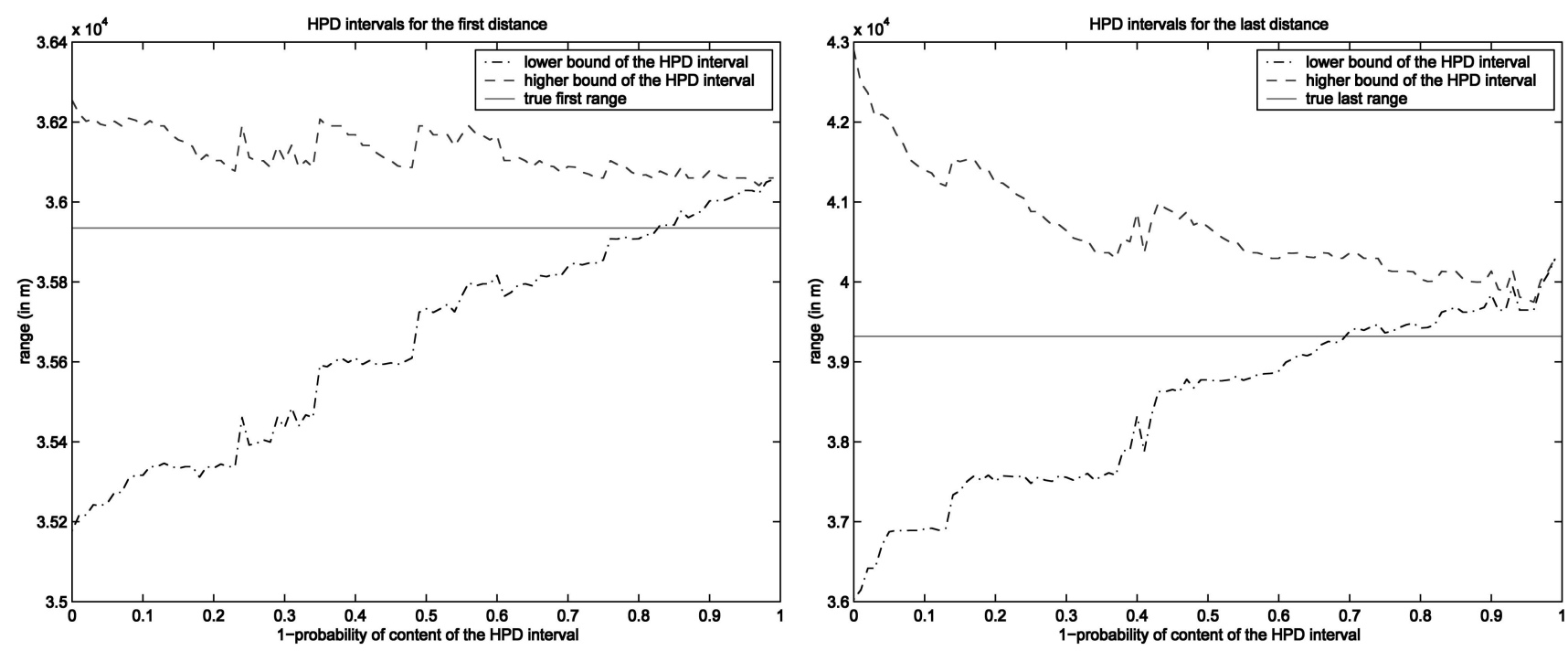

Fig. 17. Left: HPD intervals for first range $r 1$. Right: HPD intervals for third range $r 3$.

For the first leg, the target is within a small rectangle of length 3,000 m and width $1000 \mathrm{~m}$ (representing a strait for example) and the second leg is assumed to be within the larger rectangle of length $15,000 \mathrm{~m}$ and width $10,000 \mathrm{~m}$ as depicted in Fig. 16 (left). The observer is at the origin at time 0 and goes along the $x$-axis with a constant speed of 180 knots $(92.6 \mathrm{~m} / \mathrm{s})$, while the target speed is 20 knots $(10.3 \mathrm{~m} / \mathrm{s})$. The observer turns with a (very) weak angle of 2 deg after 25 observed measurements. In all, 50 bearings are taken, with $5 \mathrm{~s}$ between each measurement. The measurement error is modelled by a centered Gaussian noise, with $1 \mathrm{deg}$ standard deviation as presented in Fig. 16 (right). The target is approximately $36,000 \mathrm{~m}$ far from the observer. The total target (respectively observer) trajectory length is thus $2,524 \mathrm{~m}$ (respectively $22,678 \mathrm{~m})$.
The target trajectory is defined by 6 parameters, ${ }^{13}$ namely, ranges $\left\{r_{1}, r_{2}, r_{3}\right\}$ and bearings $\left\{\theta_{1}, \theta_{2}, \theta_{3}\right\}$. An arbitrary initial trajectory being chosen, 5000 iterations of the hit-and-run sampler provide a set of first range $r_{1}^{i}$, a set of middle range $r_{2}^{i}$ and a set of final range $r_{3}^{i}$ related to the three points $P_{\text {init }}, P_{\text {mid }}$ and $P_{\text {end }}$ defining a leg-by-leg target trajectory. In the same way, three sets of bearings are generated. It remains to apply the HPD intervals method to each of them so as to provide six sets $(r, \theta)$ of confidence intervals about these parameters. Figs. 17 and 18 (left and right) illustrate the behavior of the proposed method.

Obviously, the hit-and-run algorithm perform quite satisfactorily, even for this difficult scenario. Even if first range HPD is far smaller than the third one, the

${ }^{13}$ It is simply assumed that the target maneuver is constrained to occur in a given domain (here the intersection of two domains). 

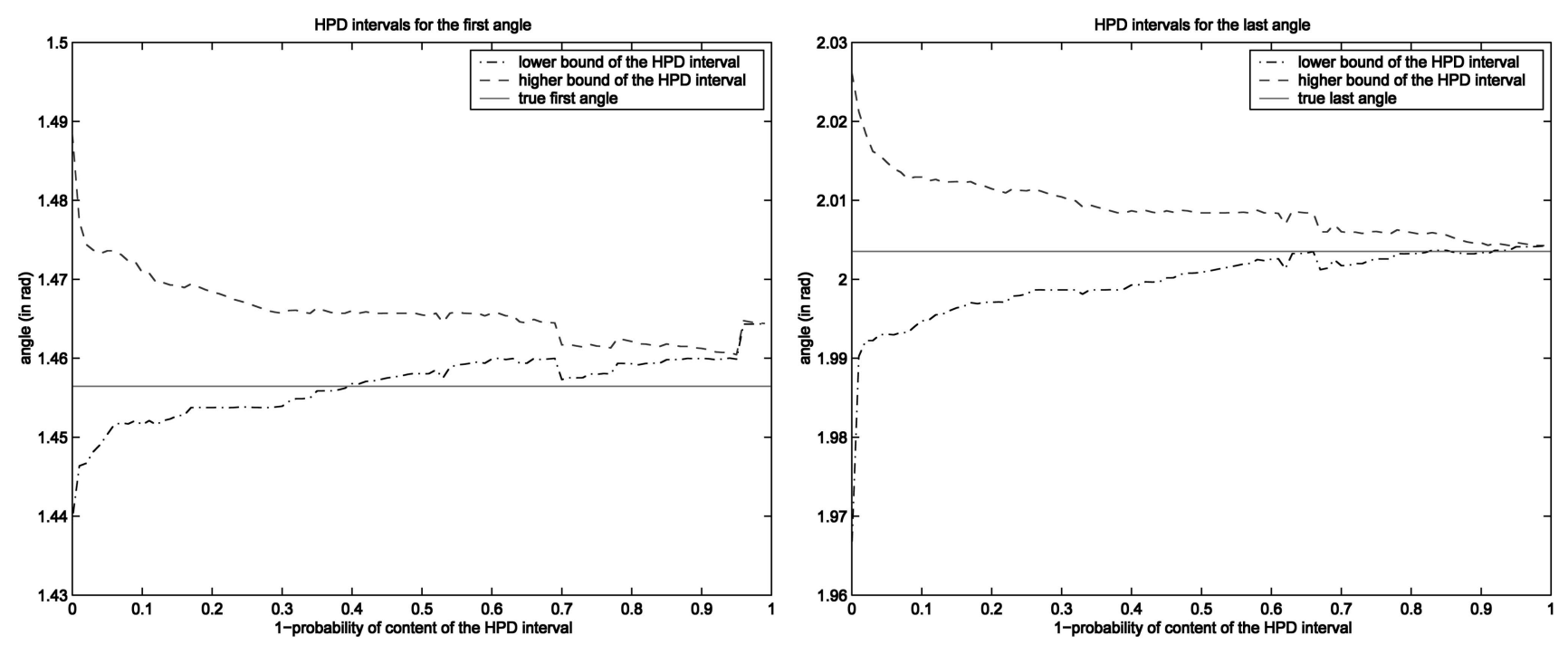

Fig. 18. Left: HPD intervals for first bearing $\theta_{1}$. Right: HPD intervals for third bearing $\theta_{3}$.

third range estimation remains quite acceptable. For the angle estimation, HPD intervals are of the same order. It is remarkable that such performance has been achieved with a very weak observer maneuver.

\section{CONCLUSION}

This paper deals with range estimation estimation under poor observability conditions via operational constraints reinforcement. The algorithms we have developed are based on simulating constrained MCMC converging toward the posterior distribution of the target trajectory. Effects of constraints have been investigated, both from a statistical perspective (Cramér-Rao bounds) and a deterministic one (intervals). Geometry of nonobservable BOT TMA has been analyzed both from a nonlinear viewpoint (the envelope geometry) and a linear one (the geometry of the observable subspace). Performance is illustrated by simulation results, showing the considerable improvements offered by these methods.

Due to an exhaustive exploration of the posterior density, these algorithms are both reliable and feasible. Using them, it is possible to exploit complex kinematic and geographic constraints on target behavior and thus to provide a convenient estimate for hardly estimable parameter(s). Moreover, reliable confidence intervals are obtained as a by-product and are a definite advantage of simulation-based methods. This is of fundamental importance from an operational viewpoint; more specifically for problems like threat assessment, sensor management, or tactical aids. From an estimation perspective, these algorithms provide a basic ground for a wide variety of problems like track initialization, track-to-track association, or deghosting.

Other important features of this approach are also particularly noticeable. First, the robustness to the choice of the initial starting point is particularly appealing. Second is the avoidance of gradient-based methods, whose definite drawback is to perform poorly as soon as the problem is poorly conditioned.

\section{ACKNOWLEDGMENTS AND DEDICATION}

The authors are greatly indebted to the reviewers and the editor for an insightful review process.

This paper is dedicated to the memory of Philippe Nicolas (Thales), our friend and colleague, who was a constant source of inspiration and enthusiasm.

\section{APPENDIX A. BOT TMA PERFORMANCE ANALYSIS (NONMANEUVERING OBSERVER)}

The aim of this appendix is to quantify the effect of the BOT TMA scenario parameters, for a nonmaneuvering observer. We refer to Fig. 1 for the meaning of the parameters ( $t$ : time index). Elementary calculations yield

$$
\begin{aligned}
\tan \left(\theta_{t}\right) & =\frac{\operatorname{det}\left(\overrightarrow{M_{0} M_{t}, \vec{M}_{t}}\right)}{\left\langle\overrightarrow{M_{0} M_{t}}, \overrightarrow{M_{t} T_{t}}\right\rangle} \\
& =\frac{\sin \theta_{0}+\left(\frac{v_{\mathrm{tgt}} \sin \alpha}{r_{0}}\right) t}{\cos \theta_{0}+\left(\frac{v_{\mathrm{tgt}} \cos \alpha-v_{\mathrm{obs}}}{r_{0}}\right) t} .
\end{aligned}
$$

We can notice that $\left(v_{\mathrm{tgt}} \sin \alpha\right)$ is the radial (relative) target velocity, while $\left(v_{\mathrm{tgt}} \cos \alpha-v_{\mathrm{obs}}\right)$ is the longitudinal one. From (28), we see that the target bearing history is described by a set of three parameters (e.g. $\left\{\theta_{0},\left(v_{\text {tgt }} \sin \alpha\right) / r, 1 / r\right\}$ or $\left\{\theta_{0}, \alpha, r^{\prime} \triangleq 1 / r\right\}$ ). This is certainly not surprising since in the absence of observer maneuvering the dimension of the observable space is generally 3 . 
Thus, estimability analysis is achieved by considering only the estimation of 3 parameters, the fourth being fixed. For this appendix, we consider the target trajectory parameterization $\left\{\theta_{0}, \alpha, r^{\prime} \triangleq 1 / r\right\}$, the parameter $v_{\text {tgt }}$ being fixed $\left(r \equiv r_{0}\right)$.

Practically, an estimability measure is obtained in the following way [27]. Let $\mathbf{g}_{t}$ be the gradient vector of the measurement, i.e., $\mathbf{g}_{t}=$ $\left\{\left(\partial / \partial \theta_{0}\right) \theta_{t},(\partial / \partial \alpha) \theta_{t},\left(\partial / \partial r^{\prime}\right) \theta_{t}\right\}^{T}$ and define the $(3 \times 3)$ $\mathbf{M}$ matrix as $\mathbf{M}=\left(\mathbf{g}_{t}, \dot{\mathbf{g}}_{t}, \ddot{\mathbf{g}}_{t}\right)$. Then, we consider as a measure of the uncertainty (also termed estimability), the determinant of the FIM, itself approximated by (three consecutive measurements: $\{0,1,2\}$, Gaussian hypothesis):

$$
\begin{aligned}
& \operatorname{det}(\mathrm{FIM}) \approx\left[\operatorname{det}\left(\mathbf{g}_{t}, \dot{\mathbf{g}}_{t}, \ddot{\mathbf{g}}_{t}\right)\right]_{t=0}^{2} \\
& \text { yielding: }
\end{aligned}
$$

$$
\begin{aligned}
\operatorname{det}\left(\mathrm{FIM}_{0,2}\right)= & \frac{4 v_{\mathrm{tg}}^{2} r^{\prime 4}}{\sigma_{w}^{6}\left(\cos \theta_{0}\right)^{12}}\left(v_{\mathrm{tgt}}-v_{\mathrm{obs}} \cos \alpha\right)^{2} \\
& \times\left(v_{\mathrm{tgt}} \sin \left(\alpha-\theta_{0}\right)+v_{\mathrm{obs}} \sin \theta_{0}\right)^{2} \\
= & \frac{4 v_{\mathrm{tg}}^{2} r^{\prime 2}}{\sigma_{w}^{6}\left(\cos \theta_{0}\right)^{12}}\left(v_{\mathrm{tgt}}-v_{\mathrm{obs}} \cos \alpha\right)^{2} \dot{\theta}^{2} \\
& \leftarrow \text { param.: }\left\{\theta_{0}, \alpha, r^{\prime}\right\} .
\end{aligned}
$$

From (29), we see that the uncertainty volume (i.e., $[\operatorname{det}(\mathrm{FIM})]^{-1}$ ) varies with $r^{2}, \dot{\theta}^{-2} \cdot{ }^{14}$ Moreover, considering the basic assumption of this manuscript (i.e., $v_{\mathrm{obs}} \gg v_{\mathrm{tgt}}$ ) and assuming $v_{\mathrm{tgt}}$ known, yields

$$
\operatorname{det}\left(\mathrm{FIM}^{-1}\right) \propto\left(\frac{r}{v_{\text {obs }}}\right)^{4} \frac{\left(\cos \theta_{0}\right)^{12}}{v_{\mathrm{tgt}}^{2}\left(\sin \theta_{0} \cos \alpha\right)^{2}}, \quad \leftarrow \text { param.: }\left\{\theta_{0}, \alpha, r^{\prime}\right\} .
$$

This formula needs some comments. First, the importance of the value of $\cos \theta_{0}$ is noteworthy (exponent: 12), as well as that of the ratio $\left(r / v_{\text {obs }}\right)$ (exponent: 4). The above analysis can be extended in two principal ways. First, for a larger number of $T$ consecutive measurements, the Binet-Cauchy formula [27] allows us to treat any measurement number, and the preceding approximations becomes

$$
\operatorname{det}\left[\operatorname{FIM}_{(1, \ldots, T)}\right] \propto\left[T^{6}\right]^{2} \operatorname{det}\left(\operatorname{FIM}_{0,2}\right) .
$$

This approximation is valid for a reasonably long baselength. Notice that the term $T^{6}$ is homogeneous with the speed factor $\left(v_{\text {tgt }}^{2} v_{\text {obs }}^{4}\right)$.

Second, let us consider a random prior about the unobservable parameter. Performance analysis will be considered now on lower bounds of the estimate variances. More precisely, let $\mathbf{x}$ be the complete state vector, while $x_{1}$ is the missing parameter (here

\footnotetext{
${ }^{14}$ The equality $r^{\prime} \dot{\theta}=v_{\text {tgt }} \sin \left(\alpha-\theta_{0}\right)+v_{\text {obs }} \sin \theta_{0}$ is obtained from elementary calculations.
}

$v_{\text {tgt }}$ or $r$ ) and consider the following decomposition $\mathbf{x}^{T}=\left(\mathbf{x}_{1}, x_{1}\right)$. For instance, consider the $\mathbf{x}_{1}=\left\{\theta_{0}, \alpha, r^{\prime}\right\}$ parametrization $\left(x_{1}=v_{\text {tgt }}\right.$ fixed), then the diagonal terms of $\mathrm{FIM}^{-1}$ have relatively simple expressions, i.e.,

$$
\begin{aligned}
& \operatorname{FIM}^{-1}(1,1)=1 \\
& \operatorname{FIM}^{-1}(2,2)= \\
& {\left[1+16 r^{\prime 2}\left(v_{\mathrm{tgt}} \cos \left(\alpha-\theta_{0}\right)-v_{\mathrm{obs}} \cos \left(\theta_{0}\right)\right)^{2}\right.} \\
& \frac{\left.+4 r^{\prime 4}\left(v_{\mathrm{obs}}^{2}+v_{\mathrm{tgt}}^{2}-2 v_{\mathrm{obs}} v_{\mathrm{tgt}} \cos \alpha\right)^{2}\right]}{4 r^{\prime 4} v_{\mathrm{tgt}}^{2}\left(v_{\mathrm{tgt}}-v_{\mathrm{obs}} \cos \alpha\right)^{2}}
\end{aligned}
$$

$\operatorname{FIM}^{-1}(3,3)=$

$$
\begin{aligned}
& {\left[\cos ^{2}\left(\alpha-\theta_{0}\right)+4 r^{\prime 2}\left(-v_{\mathrm{tgt}} \cos \left(2\left(\alpha-\theta_{0}\right)\right)+v_{\mathrm{obs}}\right.\right.} \\
& \frac{\left.\left.\cos \left(\alpha-2 \theta_{0}\right)\right)^{2}+4 r^{\prime 4}\left(v_{\mathrm{obs}}^{2}+v_{\mathrm{tgt}}^{2}-2 v_{\mathrm{obs}} v_{\mathrm{tgt}} \cos \alpha\right)^{2}\right]}{4 r^{\prime 2}\left(v_{\mathrm{tgt}}-v_{\mathrm{obs}} \cos \alpha\right)^{2}\left(v_{\mathrm{tgt}} \sin \left(\alpha-\theta_{0}\right)+v_{\mathrm{obs}} \sin \theta_{0}\right)^{2}} .
\end{aligned}
$$

Furthermore, admitting that the assumption $v_{\text {obs }} r^{\prime} \ll 1$ is reasonable, an approximation of $\operatorname{FIM}^{-1}(3,3)$ (associated with the $r^{\prime}$ estimation) becomes as simple as:

$$
\operatorname{FIM}^{-1}(3,3) \approx \frac{r^{2} \cos ^{2}\left(\alpha-\theta_{0}\right)}{4 v_{\mathrm{obs}}^{4}\left(\cos \alpha \sin \theta_{0}\right)^{2}} .
$$

Now, a classical and important result is that the following chain of (matrix) inequalities holds true [2]:

$$
\operatorname{cov}\left(\hat{\mathbf{x}}_{1}-\mathbf{x}_{1}\right) \succeq\left[\mathrm{FIM}_{\mathbf{x}_{1}}\right]^{-1} \succeq\left(\mathrm{FIM}_{\mathbf{x}}^{-1}\right)_{\overline{1}, \overline{1}} \succeq\left[\left\{\mathrm{FIM}_{\mathbf{x}}\right\}_{\overline{1}, \overline{1}}\right]^{-1}
$$

where FIM $_{\mathbf{x}_{1}}$ is the FIM matrix relative to the subvector $\mathbf{x}_{1}$ (parameter $x_{1}$ being fixed), and $\left(\text { FIM }_{\mathbf{x}}^{-1}\right)_{\overline{1}, \overline{1}}$ is the $3 \times 3$ left-upper block of the FIM $^{-1}$ matrix. Then, assuming a random prior about parameter $x_{1}$ (here $v_{\text {tgt }}$ ), whose support is denoted $\mathcal{C}_{1}$, we have:

$$
\mathbf{E}\left[\operatorname{cov}\left(\hat{\mathbf{x}}_{1}-\mathbf{x}_{1}\right)\right] \succeq \int_{\mathcal{C}_{1}}\left[\mathrm{FIM}_{\mathbf{x}_{1}}^{-1}\right]_{x_{1}=u} p_{x_{1}}(u) d u .
$$

The diagonal terms of the matrix $\left[\mathrm{FIM}_{\mathbf{x}_{1}}^{-1}\right]_{x_{1}=u}$ are given by (32). Considering for instance (33), we obtain

$$
\begin{aligned}
\operatorname{var}\left(\hat{r}^{\prime}\right) \geq & \frac{\cos ^{2}\left(\alpha-\theta_{0}\right)}{4 r^{\prime 2}} \int_{\mathcal{C}_{1}} \\
& \times \frac{p_{v_{\mathrm{tgt}}}(u)}{\left(v_{\mathrm{tgt}}-v_{\mathrm{obs}} \cos \alpha\right)^{2}\left(v_{\mathrm{tgt}} \sin \left(\alpha-\theta_{0}\right)+v_{\mathrm{obs}} \sin \theta_{0}\right)^{2}} d u .
\end{aligned}
$$

\section{APPENDIX B. GEOMETRY OF NONOBSERVABLE BOT TMA}

Throughout this appendix, our objective is to investigate the geometry of nonobservable BOT TMA, which means that it is not a statistical analysis. We are now dealing with the proof of Proposition 1. For the sake of concision, notations are slightly simplified and 


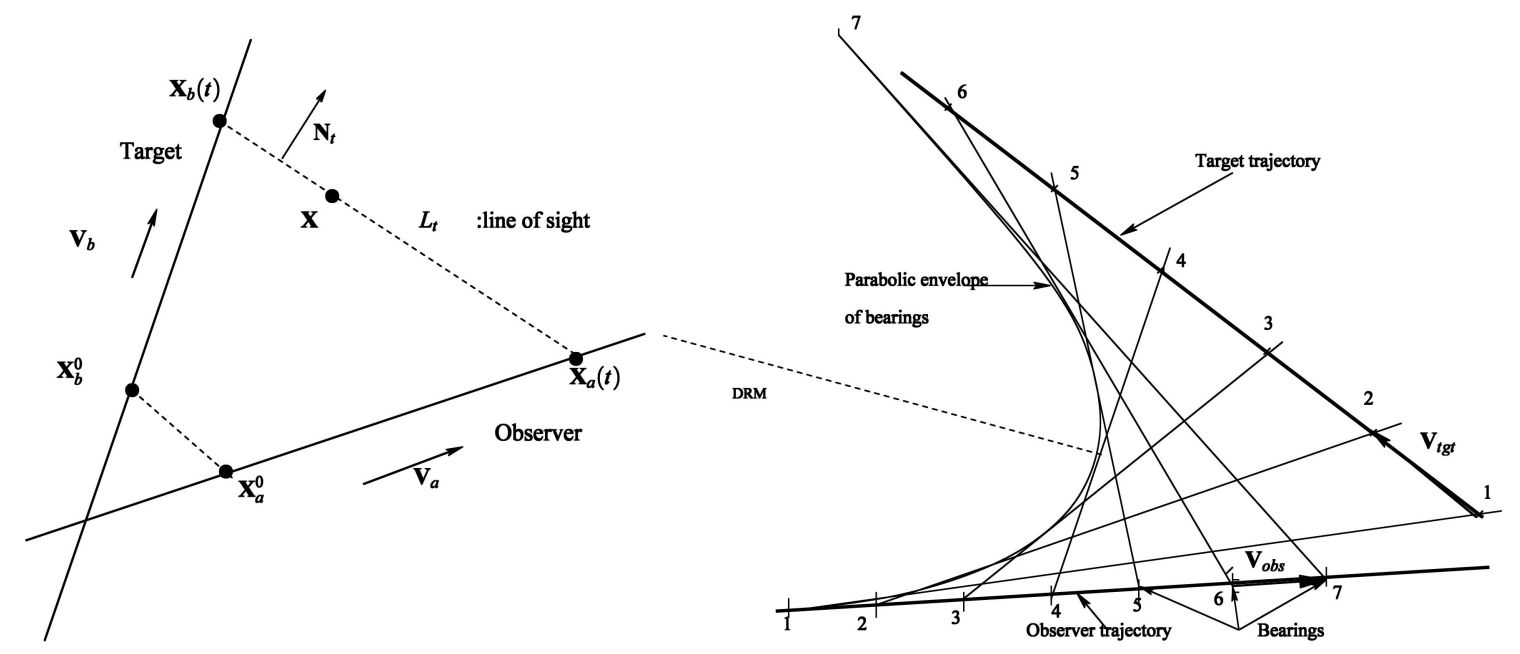

Fig. 19. Left: geometric definitions. Right: parabolic envelope of bearing lines and its axis.

are those of Fig. 19. The vector $\mathbf{N}(t)$ is orthogonal to the bearing line $L_{t}$. Thus, we can write $(Q: \pi / 2$ rotation matrix):

$$
\begin{aligned}
& \mathbf{N}(t)=Q\left[\mathbf{X}^{0}+t \mathbf{V}\right] \quad \text { where } \\
& Q=\left(\begin{array}{cc}
0 & -1 \\
1 & 0
\end{array}\right) \\
& \mathbf{X}^{0} \triangleq \mathbf{X}_{b}^{0}-\mathbf{X}_{a}^{0} \\
& \mathbf{V} \triangleq \mathbf{V}_{b}-\mathbf{V}_{a}\left(\mathbf{V}_{b} \triangleq \overrightarrow{v_{\mathrm{tgt}}}, \mathbf{V}_{a} \triangleq \overrightarrow{v_{\mathrm{obs}}}\right) .
\end{aligned}
$$

Denoting $\mathbf{X}$ the generic point of $L_{t}$, an (implicit) equation of the bearing line $L_{t}$ is then given by

$$
g(\mathbf{X}, t) \triangleq\left(\mathbf{X}-\mathbf{X}_{a}(t)\right)^{T} Q\left(\mathbf{X}^{0}+t \mathbf{V}\right)=0 .
$$

Then, the envelope of the bearing lines $\left\{L_{t}\right\}_{t}$ is defined by the following system of equations:

$$
g(\mathbf{X}, t)=0, \quad \frac{\partial}{\partial t} g(\mathbf{X}, t)=0 .
$$

From the second equation $((\partial / \partial t) g(\mathbf{X}, t)=0)$, we obtain the following condition which is necessarily satisfied by a point $\mathbf{X}$ belonging to the envelope of the $L_{t}$ lines:

$$
t=\frac{1}{2\left(\mathbf{V}_{a}^{T} Q \mathbf{V}_{b}\right)}\left[\left(\mathbf{X}-\mathbf{X}_{a}^{0}\right)^{T} Q \mathbf{V}-\mathbf{V}_{a}^{T} Q \mathbf{X}^{0}\right] .
$$

Injecting (40) in $g(\mathbf{X}, t)$, we deduce an equation of the envelope (see (39)), i.e.,

$$
\mathbf{X}^{T}\left(Q \mathbf{V} \mathbf{V}^{T} Q^{T}\right) \mathbf{X}+\alpha_{1} \mathbf{X}^{T} Q \mathbf{X}^{0}+\beta_{1} \mathbf{X}^{T} Q \mathbf{V}+\gamma_{1}=0
$$

where

$$
\begin{aligned}
& \alpha_{1}=4\left(\mathbf{V}_{a}^{T} Q \mathbf{V}_{b}\right) \\
& \beta_{1}=-2\left(\mathbf{X}_{a}^{0, T} Q \mathbf{V}+\mathbf{V}_{a}^{T} Q \mathbf{X}^{0}\right) \\
& \gamma_{1}=\left(\mathbf{X}_{a}^{0, T} Q \mathbf{V}+\mathbf{V}_{a}^{T} Q \mathbf{X}^{0}\right)^{2}+4\left(\mathbf{V}_{a}^{T} Q \mathbf{V}_{b}\right)\left(\mathbf{X}_{a}^{0, T} Q \mathbf{X}_{b}\right) .
\end{aligned}
$$

From (41), we see that the envelope of the $L_{t}$ lines is a parabola. Indeed, $g(\mathbf{X}, t)$ is a quadratic form in $\mathbf{X}$ whose associated matrix is $(Q \mathbf{V})(Q \mathbf{V})^{T}$. This matrix is nonnegative definite while its rank is 1 which proves that this envelope is a parabola (see [15], chapter $\mathrm{X})$. Its image is one-dimensional and generated by the vector $Q \mathbf{V}$, while its kernel is spanned by the vector $\mathbf{V}$ (direction of relative motion (DRM), see Fig. 19). Thus, we have just shown the first part of Proposition 1.

The second part of Proposition 1 is much more intricate. An analytic proof is feasible but seems (at best) very tedious. Instead, a purely geometric proof can be found in classical (but ancient) treatises on geometry. Reference [24] does not require any prerequisite, while a large part is devoted to conic properties. Now, let us refer to the following figure: let $\Delta(A P)$ and $\Delta(A N)$ be two fixed tangent lines to the parabola, and $\triangle(B C)$ a generic line, tangent in $M . B$ and $C$ are the intersections of this tangent line with $\Delta(A P)$ and $\Delta(A N)$. Then, with the notations of Fig. 20, the following equality holds true:

$$
\frac{M B}{M C}=\frac{B P}{B A}=\frac{C A}{C N} .
$$

Actually, this remarkable property is valid whatever the point $M$ and is a consequence of Poncelet theorems [24]. So, to end the proof it remains to prove that the target trajectory is also a tangent to the envelope parabola. This is easily achieved by considering the following equalities:

$$
\begin{aligned}
(\mathbf{X}- & \left.\mathbf{X}_{b}(t)\right)^{T} Q\left(\mathbf{X}^{0}+t \mathbf{V}\right) \\
& =g(\mathbf{X}, t)+\left(\mathbf{X}_{a}-\mathbf{X}_{b}(t)\right)^{T} Q\left(\mathbf{X}^{0}+t \mathbf{V}\right) \\
& =g(\mathbf{X}, t)+\left(\mathbf{X}^{0}+t \mathbf{V}\right)^{T} Q\left(\mathbf{X}^{0}+t \mathbf{V}\right)=g(\mathbf{X}, t) .
\end{aligned}
$$




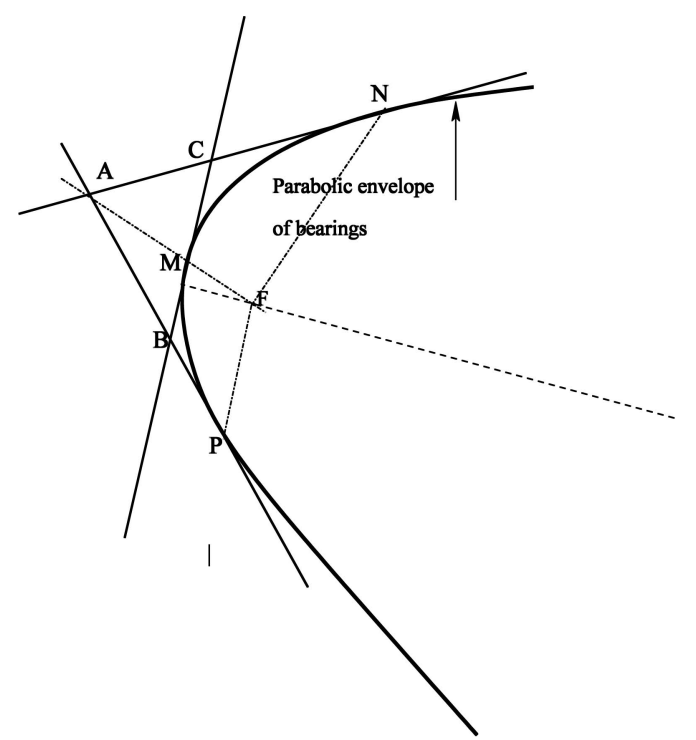

Fig. 20. Algebraic properties of the tangent lines of a parabola.

Thus, from (43), we see that target and observer play symmetric roles. Since the target trajectory is tangent to the bearing lines envelope, so is the observer trajectory and this ends the proof of Proposition 1.

Let us now consider the proof of Proposition 2. We use now the tools of (elementary) linear algebra. Assuming that both target and observer are in rectilinear and uniform motions, then bearings-angle $\theta_{i}$ are given by

$$
\tan \theta_{i}=\frac{r_{y}(0)+i v_{y}(0)}{r_{x}(0)+i v_{x}(0)}, \quad i=1, \ldots, N
$$

where $\left\{r_{x}(0), r_{y}(0), v_{x}(0), v_{y}(0)\right\}$ are the (relative) Cartesian at a reference time (here 0 ). The target trajectory may be parameterized by the 3-dimensional vector $\mathbf{Y}=\left(r_{x}(0), v_{x}(0), r_{y}(0)\right)^{T}$, so that (44) can be rewritten as the following (linear) system:

$$
\begin{aligned}
& \mathcal{B}_{\theta} \mathbf{Y}=v_{y}(0) \mathbf{t} \quad \text { where } \\
& \mathcal{B}_{\theta}=\left(\begin{array}{ccc}
\tan \theta_{1} & \tan \theta_{1} & -1 \\
\tan \theta_{2} & 2 \tan \theta_{2} & -1 \\
\vdots & \vdots & \vdots \\
\tan \theta_{N} & N \tan \theta_{N} & -1
\end{array}\right) \\
& \mathbf{t}=\left(\begin{array}{c}
1 \\
2 \\
\vdots \\
N
\end{array}\right)
\end{aligned}
$$

Let us define the set of feasible target trajectories as the set of rectilinear uniform motions which provide the set of (exact) measurements $\left\{\theta_{1}, \theta_{2}, \ldots, \theta_{N}\right\}$, as well as $\mathbf{X}=\left\{r_{x}(0), v_{x}(0), r_{y}(0), v_{y}(0)\right\}^{T}$ a 4-dimensional

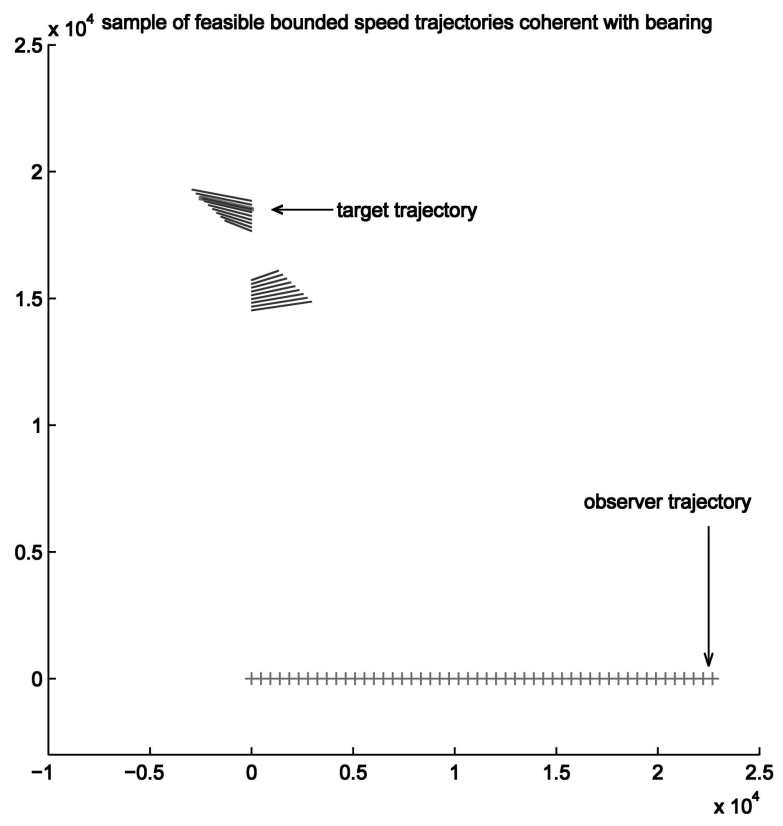

Fig. 21. Set of feasible trajectories with speed constraints $v_{\text {min }}=10$ knots $\leq v \leq v_{\text {max }}=25$ knots.

state vector, associated with the (true) target trajectory. Then, from (45):

$$
\begin{aligned}
\mathbf{X} & =\left(\begin{array}{c}
v_{y}(0) \mathcal{B}_{\theta}^{\#} \mathbf{t} \\
v_{y}(0)
\end{array}\right) \quad \text { where } \\
\mathcal{B}_{\theta}^{\#} & =\left(\mathcal{B}_{\theta}^{T} \mathcal{B}_{\theta}\right)^{-1} \mathcal{B}_{\theta} .
\end{aligned}
$$

From (46), we are now in position to represent the set of feasible trajectories. To that aim, it is sufficient to consider variations of the free parameter and to test the constraints, e.g., for speed constraints ${ }^{15} v_{\min }^{2} \leq$ $v_{x}^{2}(0)+v_{y}^{2}(0) \leq v_{\max }^{2}$. This is illustrated by Fig. 21, where the observer and target parameters are identical to that of Fig. 12, except for the target speed which is simply the opposite. The only constraints are (target) speed constraints and there are: $v_{\mathrm{tg}, \mathrm{min}}=10$ knots, $v_{\text {tgt, } \max }=25$ knots.

The set of feasible trajectories have the shape of an hourglass (see Fig. 21). It is divided in two parts, one corresponding to the "true" signs of $v_{y}(0)$ and $v_{x}(0)$ (see (46)), the other to the opposite (the "ghost" trajectories). We can notice the importance of speed constraints for reducing the area of feasible trajectories, even with rough constraints. Let us give now a more precise interpretation to the above result. First, we denote $\left(\mathcal{B}_{\theta}^{-1} \mathbf{t}\right)_{i}$ the $i$ th component of the vector $\mathcal{B}_{\theta}^{-1} \mathbf{t}, i=1,2$ or 3 . The speed constraint then stands as follows:

$$
v_{\min }^{2} \leq v_{y}^{2}\left(1+\left(\mathcal{B}_{\theta}^{-1} \mathbf{t}\right)_{2}^{2}\right) \leq v_{\max }^{2}
$$

\footnotetext{
${ }^{15}$ Note that these constraints are defined in terms of the relative speed.
} 
so, that $v_{y, \text { max }}^{2}=v_{\text {max }}^{2} /\left(1+\left(\mathcal{B}_{\theta}^{-1} \mathbf{t}\right)_{2}^{2}\right)$ and the extremal vectors of the (convex) set of feasible trajectories (see Fig. 21) are given by

$$
\begin{aligned}
r_{x, \text { max }}^{2} & =\left(\mathcal{B}_{\theta}^{-1} \mathbf{t}\right)_{1}^{2} v_{y, \text { max }}^{2} \\
r_{x, \text { min }}^{2} & =\left(\mathcal{B}_{\theta}^{-1} \mathbf{t}\right)_{1}^{2} v_{y, \text { min }}^{2} \\
r_{y, \text { max }}^{2} & =\left(\mathcal{B}_{\theta}^{-1} \mathbf{t}\right)_{3}^{2} v_{y, \text { max }}^{2} \\
r_{x, \text { min }}^{2} & =\left(\mathcal{B}_{\theta}^{-1} \mathbf{t}\right)_{3}^{2} v_{y, \text { min }}^{2} .
\end{aligned}
$$

From (48), we deduce straightforwardly: ${ }^{16}$

$$
\begin{aligned}
\left\|\vec{r}_{\max }\right\|^{2}-\left\|\vec{r}_{\min }\right\|^{2} & =\left(v_{\max }^{2}-v_{\min }^{2}\right) \frac{\left(\mathcal{B}_{\theta}^{-1} \mathbf{t}\right)_{1}^{2}+\left(\mathcal{B}_{\theta}^{-1} \mathbf{t}\right)_{3}^{2}}{1+\left(\mathcal{B}_{\theta}^{-1} \mathbf{t}\right)_{2}^{2}} \\
& =\left(v_{\max }^{2}-v_{\min }^{2}\right)\left(\frac{r^{2}}{v^{2}}\right) .
\end{aligned}
$$

This result is surprisingly simple. The effect of speed constraints $\left(v_{\max }^{2}-v_{\min }^{2}\right)$ as well as scenario parameters on the ratio $\left(r^{2} / v^{2}\right)$ is noteworthy. Notice also that the ratio $r^{2} / v^{2}$ is independent of the the particular choice of the "free" parameter $v_{y}(0)$ (see (46)). Hence, it is well defined.

From an operational viewpoint, this result is particularly enlightening. Actually, it is worth to simply rewrite it as

$$
\left\|\vec{r}_{\max }\right\|^{2}-\left\|\vec{r}_{\min }\right\|^{2}=\frac{\left(v_{\max }^{2}-v_{\min }^{2}\right)}{v^{2}} r^{2}
$$

and to examine the ratio $\left(v_{\max }^{2}-v_{\min }^{2}\right) / v^{2}$. Invoking the validity of the assumption $v_{\mathrm{tgt}} \ll v_{\mathrm{obs}}$, we have

$$
\frac{\left(v_{\max }^{2}-v_{\min }^{2}\right)}{v^{2}} \approx 2 \frac{\left(v_{\mathrm{tgt}, \max }-v_{\mathrm{tgt}, \min }\right) \cos (\alpha)}{v_{\mathrm{obs}}} .
$$

Thus, this ratio acts as a "reduction" factor for estimating the distance $r$, under speed constraints; thus showing that the benefit of including hard constraints for range estimation is generally quite noticeable.

It remains to show that the set of feasible trajectories is a convex set. Actually, this results immediately from the norm triangular inequality. Proving that (48) corresponds to extremal vectors is a direct consequence of the fact that $v_{y, \max }^{2}=v_{\max }^{2} /$ $\left(1+\left(\mathcal{B}_{\theta}^{-1} \mathbf{t}\right)_{3}^{2}\right)$ is an extremal value of the $v_{y}^{2}$ velocities, which is itself resulting from (47).

Let us consider now the feasible set of target trajectories (see Fig. 5). From the previous results, the relative velocity vector $(\vec{v})$ for a candidate target trajectory is given by

$$
\vec{v}=\vec{v}_{\mathrm{tgt}}-\vec{v}_{\mathrm{obs}}=v_{y}\left(\begin{array}{c}
\left(\mathcal{B}_{\theta}^{-1} \mathbf{t}\right)_{2} \\
1
\end{array}\right) .
$$

\footnotetext{
${ }^{16}$ Elementary calculations yield: $\left(\mathcal{B}_{\theta}^{-1} \mathbf{t}\right)_{1}^{2}=r_{x}^{2}(0) / v_{y}^{2},\left(\mathcal{B}_{\theta}^{-1} \mathbf{t}\right)_{2}^{2}=$ $v_{x}^{2} / v_{y}^{2},\left(\mathcal{B}_{\theta}^{-1} \mathbf{t}\right)_{3}^{2}=r_{y}^{2}(0) / v_{y}^{2}$.
}

The scalar $\left(\mathcal{B}_{\theta}^{-1} \mathbf{t}\right)_{2}$ only depends of the scenario, i.e., it is common to any choice of the $v_{y}$ parameter). Thus, we have

$$
\frac{v_{y, \text { max }}^{2}}{v_{x, \text { max }}^{2}}=\frac{1}{\left(\mathcal{B}_{\theta}^{-1} \mathbf{t}\right)_{2}^{2}}=\frac{v_{y, \text { min }}^{2}}{v_{x, \text { min }}^{2}} .
$$

This equation is completed by a norm one, completely similar to (50), i.e.,

$$
v_{y, \text { max }}^{2}-v_{y, \text { min }}^{2}=\left(v_{\max }^{2}-v_{\min }^{2}\right)\left(\frac{v_{y}^{2}}{v^{2}}\right) .
$$

Gathering (53) and (54), we deduce finally:

$$
\begin{aligned}
\frac{v_{y, \text { max }}^{2}}{v_{y, \text { min }}^{2}} & =1+\frac{\left(v_{\max }^{2}-v_{\min }^{2}\right)}{v_{y, \text { min }}^{2}}\left(\frac{v_{y}^{2}}{v^{2}}\right), \\
& \approx 1+2 \frac{\left(v_{\mathrm{tgt}, \text { max }}-v_{\mathrm{tgt}, \min }\right) \cos (\alpha)}{v_{\mathrm{obs}}}\left(\frac{v_{y}^{2}}{v_{y, \text { min }}^{2}}\right)
\end{aligned}
$$

and

$$
\left(\begin{array}{c}
v_{\mathrm{tg}, \text { max }}^{x} \\
v_{\mathrm{tgt}, \text { max }}^{y}
\end{array}\right) \approx\left(\begin{array}{c}
v_{\mathrm{tgt}, \text { min }}^{x} \\
v_{\mathrm{tgt}, \text { min }}^{y}
\end{array}\right)+\beta\left(\begin{array}{c}
v_{\mathrm{obs}}^{x} \\
v_{\mathrm{obs}}^{y}
\end{array}\right)
$$

where the scalar $\beta$ is an appropriate factor, such that speed constraints remain satisfied.

\section{APPENDIX C. CONVEXITY OF THE SET OF FEASIBLE TRAJECTORIES}

The aim of this appendix is to prove the convexity of feasible trajectories and associated propositions (Propositions 3 and 4). First, let us define trajectories

$$
\mathcal{T}_{1}=\left(\begin{array}{c}
P_{\text {init }}^{1} \\
P_{\text {end }}^{1}
\end{array}\right) \quad \text { and } \quad \mathcal{T}_{2}=\left(\begin{array}{c}
P_{\text {init }}^{2} \\
P_{\text {end }}^{2}
\end{array}\right) .
$$

Then, from the definition of $S$, we have

$$
\begin{aligned}
& \mathcal{T}_{1} \in S \Longleftrightarrow\left\{\begin{array}{l}
P_{\text {init }}^{1} \in C \\
P_{\text {end }}^{1} \in C \\
\left\|P_{\text {init }}^{1}-P_{\text {end }}^{1}\right\| \leq L
\end{array}\right. \\
& \mathcal{T}_{2} \in S \Longleftrightarrow\left\{\begin{array}{l}
P_{\text {init }}^{2} \in C \\
P_{\text {end }}^{2} \in C \\
\left\|P_{\text {init }}^{2}-P_{\text {end }}^{2}\right\| \leq L .
\end{array}\right.
\end{aligned}
$$

Let $u \in[0,1]$ and let $\mathcal{T}_{3}=(1-u) \cdot \mathcal{T}_{1}+u \cdot \mathcal{T}_{2}$, associated with the trajectory $\left(\begin{array}{ll}P_{\text {init }}^{3} & P_{\text {end }}^{3}\end{array}\right)$. We show that $\mathcal{T}_{3} \in S$, where

$$
\mathcal{T}_{3}=\left(\begin{array}{c}
(1-u) \cdot P_{\mathrm{init}}^{1}+u \cdot P_{\mathrm{init}}^{2} \\
(1-u) \cdot P_{\mathrm{end}}^{1}+u \cdot P_{\mathrm{end}}^{2}
\end{array}\right) .
$$


Indeed, as $C$ is convex and $P_{\text {init }}^{1}, P_{\text {init }}^{2}, P_{\text {end }}^{1}$ and $P_{\text {end }}^{2} \in C$ we have

$$
\begin{aligned}
& P_{\text {init }}^{3}=(1-u) \cdot P_{\text {init }}^{1}+u \cdot P_{\text {init }}^{2} \in C \\
& P_{\text {end }}^{3}=(1-u) \cdot P_{\text {end }}^{1}+u \cdot P_{\text {end }}^{2} \cdot \in C .
\end{aligned}
$$

Then it remains to show that $\left\|P_{\text {init }}^{3}-P_{\text {end }}^{3}\right\| \leq L$, but

$$
\begin{aligned}
\left\|P_{\text {init }}^{3}-P_{\text {end }}^{3}\right\| & =\|(1-u) \cdot\left(P_{\text {init }}^{1}-P_{\text {end }}^{1}\right)+u \cdot\left(P_{\text {init }}^{2}-P_{\text {end }}^{2} \|\right. \\
& \leq(1-u) \cdot\left\|P_{\text {init }}^{1}-P_{\text {end }}^{1}\right\|+u \cdot\left\|P_{\text {init }}^{2}-P_{\text {end }}^{2}\right\| \leq L .
\end{aligned}
$$

\section{REFERENCES}

[1] Aidala, V. J., and Hammel, S. E. Utilization of modified polar coordinates for bearings-only tracking. IEEE Transactions on Autmatic Control, AC-28, 3 (Mar. 1983), 283-294.

[2] Bobrosky, B. Z., Mayer-Wolf, E., and Zakai, M. Some classes of global Cramér-Rao bounds. The Annals of Statistics, 15, 4 (1987), 1421-1438.

[3] Boneh, A., and Golan, A.

Constraints redundancy and feasible region boundedness by random feasible point generator (RFPG).

Presented at the Third European Congress on Operations Research, EURO III, The Annals of Statistics, Amsterdam, 1979.

[4] Broman, V., and Shensa, M. J. Bearings tracking of targets with unobservable states. In Proceedings of the American Control Conference (ACC), June 1986, 1850-1854.

[5] Belisle, C. J. P., Romeijn, H. E., and Smith, R. L. Hit-and-run algorithms for generating multivariate distributions.

Mathematics of Operations Research, 18 (1993), 255-266.

[6] Chan, Y. T., and Rudnicki, S. W.

Bearings-only and Doppler-bearing tracking using instrumental variables.

IEEE Transactions on Aerospace and Electronic Systems, 28, 4 (Oct. 1992), 1076-1083.

[7] Chen, M-H., and Schmeiser, B. W. General hit-and-run Monte Carlo sampling for evaluating multidimensional integrals.

Operations Research Letters, 19 (1996), 161-169.

[8] Chen, M-H., and Shao, Q-M.

Monte Carlo estimation of Bayesian credible and HPD intervals.

Journal of Computational and Graphical Statistics, 8 (1999), 69-92.

[9] Chen, M-H., Shao, Q-M., and Ibrahim, J. G.

Monte Carlo Methods in Bayesian Computation.

New York: Springer-Verlag, corrected second printing 2002.

[10] Doucet, A., Godsill, S. J., and Robert, C. P

Marginal maximum a posteriori estimation using Markov chain Monte Carlo.

Statistics and Computing, 12 (2002), 77-84.

[11] Everett, N., Tham, S. T., and Salmond, D. A novel MCMC tracker for stressing scenarios. In O. Drummond (Ed.), Proceedings of the SPIE, Signal and Data Processing of Small Targets, vol. 5428, 2004.

[12] Gauss, K. F

Theoria Motus Corporium Coelestium.

Boston: Little, Brown \& Co., 1857.
[13] Gelfand, A. E., and Smith, A. F. M.

Sampling based approaches to calculating marginal densities.

Journal of the American Statistical Association, 85 (1990), 398-409.

[14] Gong, K. F., Lindgren, A. G., and Nardone, S. C.

Passive localization and motion analysis with a state parameter constraint.

In Proceedings of the 15th Asilomar Conference on Circuits, Systems and Computers, Nov. 9-11, 1981, 366-374.

[15] Greub, W.

Linear Algebra (4th ed.).

New York: Springer-Verlag, 1975.

[16] Grossman, N

The Sheer Joy of Celestial Mechanics.

Boston: Birkhauser, 1996.

[17] Häggström, O.

Finite Markov Chains and Algorithmic Application. London: Cambridge University Press, Cambridge, London Mathematical Society, Student Text 52, 2002.

[18] Hastings, W. K.

Monte Carlo sampling methods using Markov chains and their applications.

Biometrika, 57 (1970), 97-109.

[19] Holtsberg, A.

A statistical analysis of bearings-only tracking. Ph.D. dissertation, Dept. of Math. Statistics, Lund Institute of Technology, 1992.

[20] Jauffret, C., and Pillon, D.

Observability in passive target motion analysis.

IEEE Transactions on Aerospace and Electronic Systems, 32, 4 (Oct. 1996), 1290-1300.

[21] Jauffret, C.

Observabilité et inversibilité de la matrice de Fisher dans les problèmes de régression non-linéaire.

Actes du XVII-eme colloque GRETSI, Vannes, France, Sept. 1999, 685-688.

[22] Kass, R. E., and Vos, P. W.

Geometrical Foundations of Asymptotic Inference. New York: Wiley, 1997.

[23] Kaufman, D. E., and Smith, R. L. Optimal direction choice for Hit-and-Run sampling. Operations Research, 46 (1998), 84-95.

[24] Lebossé, C. and Hémery, C.

Géométrie, classe de mathématiques. Programme de 1945.

Fernand Nathan 1955, Jacques Gabay (reprint, 1997).

[25] Le Cadre, J. P., and Jauffret, C.

Discrete-time observability and estimability for bearings-only target motion analysis.

IEEE Transactions on Aerospace and Electronic Systems, 33, 1 (Jan. 1997), 178-201.

[26] Le Cadre, J. P., and Jauffret, C.

On the convergence of iterative methods for bearings-only tracking.

IEEE Transactions on Aerospace and Electronic Systems, 35, 3 (July 1999), 801-817.

[27] Le Cadre, J. P

Properties of estimability criteria for target motion analysis.

IEE Proceedings on Radar, Sonar Navigation, 145, 2 (Apr. 1998), 92-99.

[28] Nardone, S. C., Lindgren, A. G., and Gong, K. F.

Fundamental properties and performance of conventional bearings-only target motion analysis.

IEEE Transactions on Automatic Control, AC-29, 9 (Sept. 1984), 775-787. 
[29] Mallick, M., Maskell, S., Kirubrajan, T., and Gordon, N. Littoral target tracking using particle filter. In Proceedings of the Fifth International Conference on Information Fusion, Annapolis, MD, July 8-11, 2002, 935-942.

[30] McCabe, B. J.

Accuracy and tactical implications of bearings-only ranging algorithms.

Operations Research, 33 (1985), 94-103.

[31] Nardone, S. C., and Graham, M. L.

A closed-form solution to bearings-only target motion analysis.

IEEE Journal of Oceanic Engineering, 22, 2 (Jan. 1997), 168-178.

[32] Nardone, S. C., and Aidala, V. J.

Observability criteria for bearings-only target motion analysis.

IEEE Transactions on Aerospace and Electronic Systems, AES-17, 2 (Mar. 1981), 162-166.

[33] Passerieux, J. M.

Estimation par régions de confiance en trajectographie passive.

Actes du XV-ème colloque GRETSI, Juan-les-Pins, Sept. 1995, 153-1156.

[34] Poor, H. V.

An Introduction to Signal Detection and Estimation (2nd ed.).

New York: Springer-Verlag, 1998.

[35] Robert, C. P., and Casella, G.

Monte Carlo Statistical Methods.

New York: Springer, Texts in Statistics, 2000.
[36] Runnals, A., and Sirlantzis, K.

Low-observable maritime tracking using Monte Carlo Markov chain methods.

In IEE Colloquium on Algorithms for Target Tracking, 1995.

[37] Siffredi, E.

Transformations sur les systèmes dynamiques. Modélisation et estimation de la cinématique d'un mobile dans le plan à partir de mesures angulaires bruitées et échantillonnées. Thèse de doctorat de 3-ème cycle, Marseille, Janvier 1982.

[38] Smith, R. L.

Monte Carlo procedures for generating random feasible solutions to mathematical programming. In Proceedings of ORSA/TIMS Conference, Washington, D.C., 1980.

[39] Smith, R. L.

Efficient Monte Carlo procedures for generating points uniformly distributed over bounded regions. Operations Research, 32 (1984), 1296-1308.

[40] Tanner, M. A.

Tools for Statistical Inference (3rd ed.).

New York: Springer-Verlag, 1996.

[41] Tierney, L.

Markov chains for exploring posterior distributions (with discussion).

Journal of the American Statistical Association, 82 (1987), $528-550$.

[42] Wagner, D. H., Mylander, W. C., and Sanders, T. J. Naval Operations Analysis, (3rd ed.).

Annapolis, MD: Naval Institute Press, 1999. 

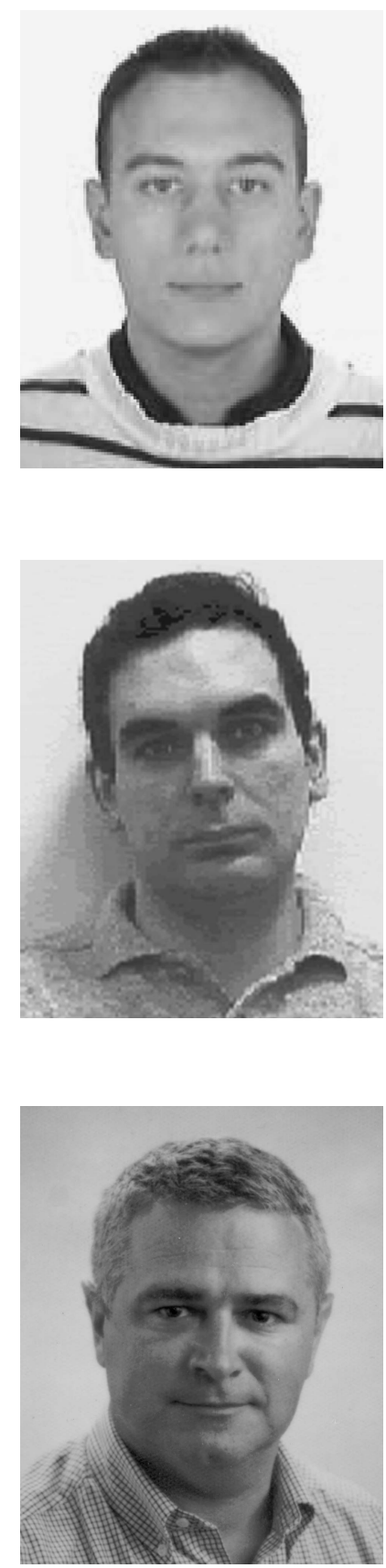

Frédéric Bavencoff received an M.S. in statistics from the Lille 1 University, France, in 2001, and the Ph.D. from the University of Rennes, France, in 2005.

$\mathrm{He}$ is with Thales Airborne Systems, France, where he is involved in performance analysis of multitarget tracking algorithms, Bayesian methods, and the theory of evidence.

Jean-Michel Vanpeperstraete graduated from ENSTA (Ecole Nationale Supérieure des Techniques Avancées), Paris, in 1986.

He began his career as an engineer with EDF (Electricité de France), and is now with Thales Airborne Systems, France, where he is involved in information processing, with a particular emphasis on tactical situation assessment.

Jean-Pierre Le Cadre (M'93) received the M.S. degree in mathematics in 1977, the "Doctorat de $3^{- \text {eme }}$ cycle" in 1982, and the "Doctorat d'Etat" in 1987, both from INPG, Grenoble.

From 1980 to 1989, he worked at the GERDSM (Groupe d'Etudes et de Recherche en Detection Sous-Marine), a laboratory of the DCN (Direction des Constructions Navales), mainly on array processing. Since 1989, he is with IRISA/CNRS, where he is "Directeur de Recherche" at CNRS. His interests are now topics like system analysis, detection, multitarget tracking, data association, and operations research.

Dr. Le Cadre has received (with O. Zugmeyer) the Eurasip Signal Processing best paper award (1993). 